\title{
Achieving High Performance on Web-Based \\ J2EE Application Servers
}

\author{
by
}

\begin{abstract}
Ala Awad, B.C.S.S.
A thesis submitted to the Faculty of Graduate Studies and Research in partial fulfillment of the requirements for the degree of
\end{abstract}

\section{Master of Applied Science in Electrical Engineering}

\author{
Ottawa-Carleton Institute for Electrical and Computer Engineering \\ Department of Systems and Computer Engineering \\ Faculty of Engineering \\ Carleton University \\ Ottawa, Ontario, Canada, K1S 5B6
}

November 2006

C Copyright 2006, Ala Awad 


$\begin{array}{ll}\begin{array}{l}\text { Library and } \\ \text { Archives Canada }\end{array} & \begin{array}{l}\text { Bibliothèque et } \\ \text { Archives Canada }\end{array} \\ \begin{array}{l}\text { Published Heritage } \\ \text { Branch }\end{array} & \begin{array}{l}\text { Direction du } \\ \text { Patrimoine de l'édition }\end{array} \\ \begin{array}{l}\text { 395 Wellington Street } \\ \text { Ottawa ON K1A ON4 }\end{array} & \begin{array}{l}\text { 395, rue Wellington } \\ \text { Ottawa ON K1A ON4 } \\ \text { Canada }\end{array}\end{array}$

Your file Votre référence ISBN: 978-0-494-23325-2 Our file Notre référence ISBN: 978-0-494-23325-2

NOTICE:

The author has granted a nonexclusive license allowing Library and Archives Canada to reproduce, publish, archive, preserve, conserve, communicate to the public by telecommunication or on the Internet, loan, distribute and sell theses worldwide, for commercial or noncommercial purposes, in microform, paper, electronic and/or any other formats.

The author retains copyright ownership and moral rights in this thesis. Neither the thesis nor substantial extracts from it may be printed or otherwise reproduced without the author's permission.
AVIS:

L'auteur a accordé une licence non exclusive permettant à la Bibliothèque et Archives Canada de reproduire, publier, archiver, sauvegarder, conserver, transmettre au public par télécommunication ou par l'Internet, prêter, distribuer et vendre des thèses partout dans le monde, à des fins commerciales ou autres, sur support microforme, papier, électronique et/ou autres formats.

L'auteur conserve la propriété du droit d'auteur et des droits moraux qui protège cette thèse. $\mathrm{Ni}$ la thèse ni des extraits substantiels de celle-ci ne doivent être imprimés ou autrement reproduits sans son autorisation.
In compliance with the Canadian

Privacy Act some supporting forms may have been removed from this thesis.

While these forms may be included in the document page count, their removal does not represent any loss of content from the thesis.
Conformément à la loi canadienne sur la protection de la vie privée, quelques formulaires secondaires ont été enlevés de cette thèse.

Bien que ces formulaires aient inclus dans la pagination, il n'y aura aucun contenu manquant.

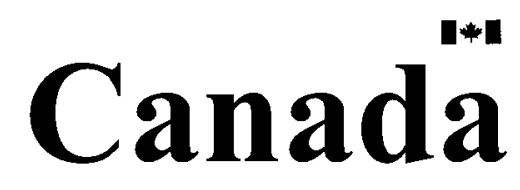




\begin{abstract}
Underlying much of the successful technology on the Web is Java- specifically the Java 2 Enterprise Edition (J2EE) application environment [5]. J2EE created an environment that allows for more focus on business logic implementation, while facilitating necessary distributed programming architecture (i.e. Enterprise JavaBeans), and common services. However, server performance handling in such a diverse Web client-based environment is of key importance. A clear understanding of system state, and adapting to it at run time, is required to achieve improved performance.
\end{abstract}

Using a publicly available J2EE application server implementation, a new Web-tierbased "Dynamic Bean Pool Resource Management" (DBPRM) technique has been devised. A prototype of the system has been implemented and studied, for improved EJB pool resource management that adapts to the system state. A performance analysis of this technique provides insights into system behavior and performance. Further studies and suggested recommendations for improvement are also presented in this work. 


\section{Acknowledgments}

I would like to express my sincere thanks to my supervisor Professor Shikharesh Majumdar for his invaluable patience, support and guidance during this thesis effort.

Further, I would like to express my deep appreciation and thanks to my wife, May, my parents Walid and Kalthoum, and my dear brother Dia, for their endless patience, understanding and strong support without which the research would not have been possible.

Last but not least, I would like to thank my friends, Amr and Hazem for their constant support and encouragement. 


\section{Table of Contents}

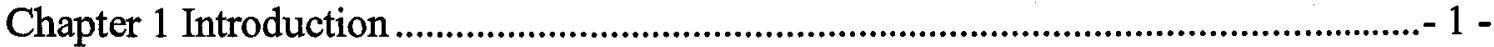

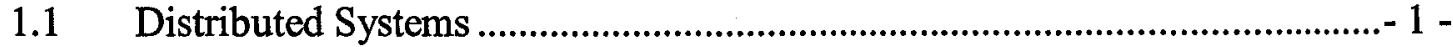

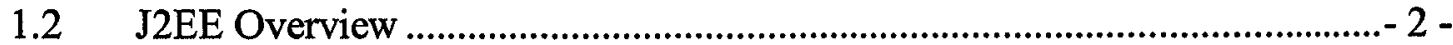

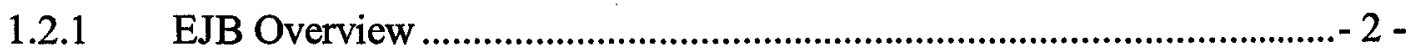

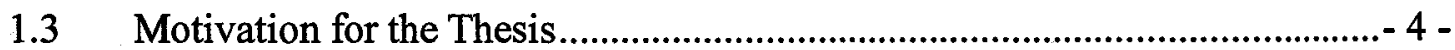

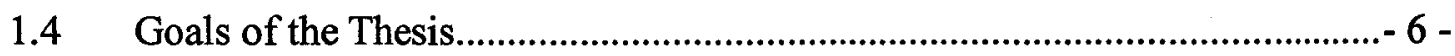

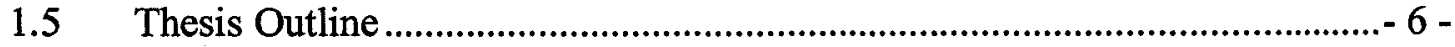

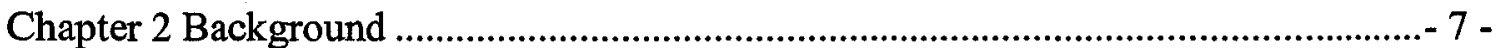

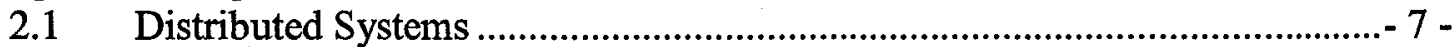

2.1.1 Challenges in Distributed Systems ...................................................... 8 -

2.2 Programming Models for Distributed Systems............................................ 11 -

2.2.1 Examples of Existing Techniques of Distributed Applications............. 12 -

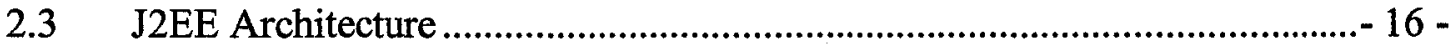

2.3.1 Presentation Tier ............................................................................. 21 -

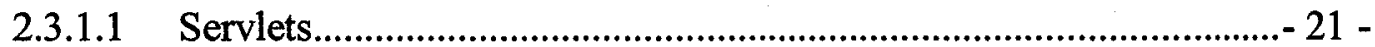

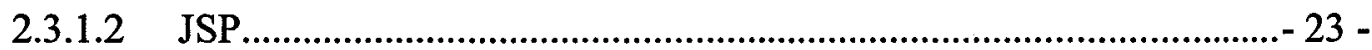

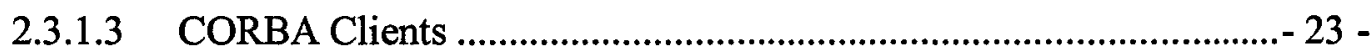

2.3.2 Logical Tier........................................................................... $24-$

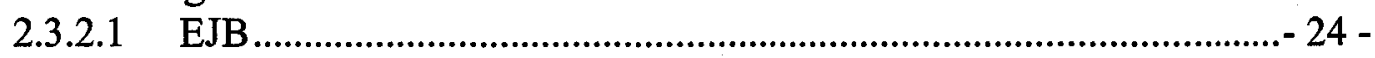

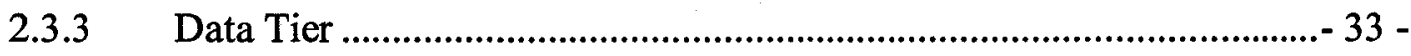

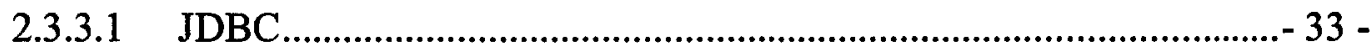

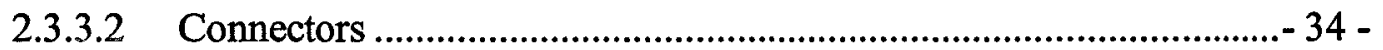

2.3.4 How has EJB Addressed Distributed Systems Challenges? .................. 35 -

2.3.4.1 Heterogeneity ............................................................................... 35 -

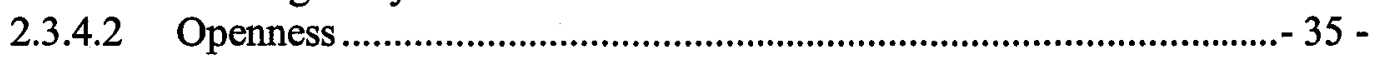

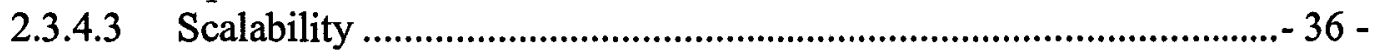

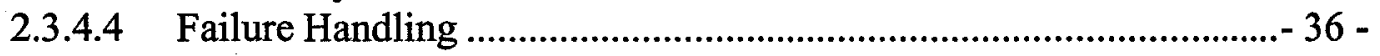

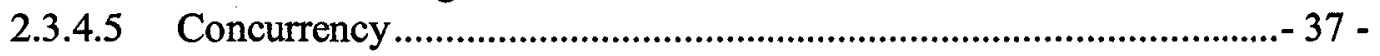

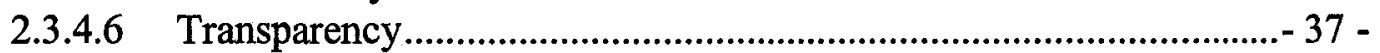

2.3.5 Configuration challenge for improving enterprise system performance - 38

2.3.5.1 System configuration challenge of Enterprise applications............... 38 -

2.3.5.2 Addressing the software configuration challenge........................... 40 -

2.3.5.3 J2EE application server configuration .......................................... 42 -

2.3.5.4 The EJB pool configuration challenge............................................. 43 -

Chapter 3 Dynamic Bean Pool Resource Management (DBPRM) ............................. 45 -

3.1 DBPRM Solution Architecture ................................................................. 45 -

3.2 DBPRM's Adopted Session Façade Pattern ................................................ 46 -

3.2.1 Definition of Session Façade Pattern .................................................. 47 -

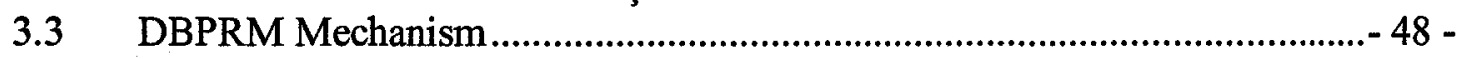

3.3.1 DBPRM's Bean-Pool-Configuration and Algorithm ......................... 51 -

Chapter 4 Experiments Conducted ................................................................ 56 -

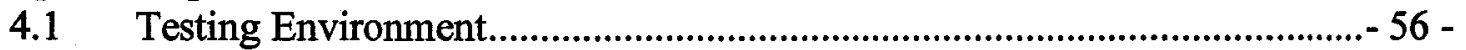

4.1.1 URL Performance Analyzer Tool .................................................... 56 - 
4.2 Results of Experiments ..............................................................................-60 -

4.2.1 Common Parameters for Performed Experiments ................................... 60 -

4.2.2 The effect of pool size on client response time..................................... 61 -

4.2.3 The Effect of Client Request Rate $(\lambda)$ on Client Response Time, with

Unequal Maximum Bean Pool Sizes for a Façade's EJBs ................................... 63 -

4.2.4 The Effect of Client Request Rate ( $\lambda$ ) with Equal Maximum Bean Pool

Sizes -66 -

4.2.5 The Effect of Client Request Rate $(\lambda)$ with Different Service Times for

EJBs - 69 -

4.2.6 The Effect of the DBPRM Over-use Time Threshold

$70-$

4.2.7 The Effect of Service Time for Façade's EJBs, while DBPRM Activated .-

$72-$

4.2.8 The Effect of Client Request Probability on Client Response Time ....- 74 -

4.2.9 The Effect of DBPRM Under-use Time Threshold ................................. 76 -

4.2.10 The Effect of Static Maximum Pool Size, versus the Effect of DBPRM's

Dynamic Adaptation to Maximum Pool Size.............................................................- 78 -

4.2.11 The Effect of DBPRM Increase/Decrease Factors ................................ 82 -

4.2.12 The Effect of a Low Client Request Rate ............................................... 85 -

Chapter 5 Conclusions ................................................................................................ 87 -

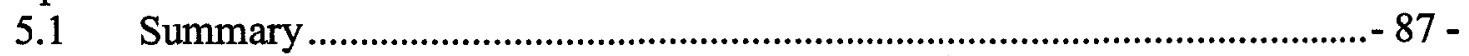

5.2 Insights Gained .................................................................................... 87 -

5.3 Thesis Contributions .............................................................................. 89 -

5.4 Future Work ..............................................................................................

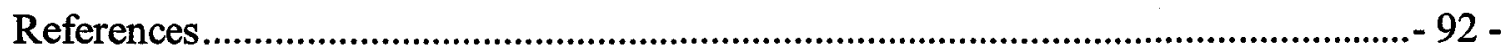

Appendix A: Detailed Design of the URL Performance Analyzer................................... 97 - 


\section{List of Figures}

Figure 1: Number of hosts on the Internet [35] ....................................................... 9 -

Figure 2: A request passing from a client to an object implementation [20].................- 14 -

Figure 3: Example of a Web-based J2EE application [26] ....................................... 18 -

Figure 4: Example of a three-tier architecture with EJB as middleware ....................... 22 -

Figure 5: The enterprise bean components [30] ...................................................... 26-

Figure 6: Retrieval of the home interface and generation of a remote interface instance [30]

Figure 7: Handling a client request to a business method [30] .................................. 29 -

Figure 8: Management of enterprise applications according to a common life cycle [16].. 39 -

Figure 9: DBPRM operational architecture $-49-$

Figure 10: DBPRM Dynamic Adaptation Algorithm............................................... 55 -

Figure 11: URL Performance Analyzer Design Architecture...................................... 58 -

Figure 12: The effect of maximum pool size on client response time.......................... 62 -

Figure 13: Effect of $\lambda$ on response time with different pool sizes in a façade's EJBs..- 65 -

Figure 14: Effect of $\lambda$ on response time with equal maximum pool sizes for the façade's

EJBs -67 -

Figure 15: Effect of client request rate $(\lambda)$ on client's response time, with exponentially

distributed service times for façade's EJBs $-68-$

Figure 16: The effect of $\lambda$ on response time with different fixed service time configurations for a façade's EJBs $-70-$

Figure 17: Effect of DBPRM over-use time threshold on client response time $-71-$

Figure 18: Effect of fixed service time on client response time, with DBPRM activated...73 -

Figure 19: Effect of client request probability on client response time. $-75-$

Figure 20: Effect of DBPRM under-use time threshold on client response time .......... 77 -

Figure 21: Comparison between a statically configured system and a DBPRM activated system. $-80-$

Figure 22: The effect of DBPRM's dynamic adaptation of maximum pool size (service time is fixed) $-81-$

Figure 23: The effect of DBPRM's dynamic adaptation of maximum pool size (service time is exponentially distributed). $-82-$

Figure 24: Effect of DBPRM Increase/Decrease Factors on Client Mean Response Time 84 -

Figure 25: Effect of low client request rate $(\lambda)$ on client response time $86-$

Figure 26: URL Performance Analyzer UML Class Model.... $-97-$ 


\section{List of Tables}

Table 1: Additional fixed parameters for the effect of fixed service time on response time, with DBPRM activated experiment ...............................................................- 73 Table 2: Additional fixed parameters for the effect of client request probability on client response time experiment $-75-$

Table 3: Additional fixed parameters for the Comparison between a statically configured system and a DBPRM activated system experiment ............................................ 79 -

Table 4: Additional fixed parameters for the effect of DBPRM Increase/Decrease Factors on Client Mean Response Time experiment ......................................................... 83 - 


\section{Glossary of Terms}

API Application Programming Interface

$\begin{array}{ll}\text { ASP } & \text { Active Server Pages } \\ \text { BMP } & \text { Bean Managed Persistence } \\ \text { CGI } & \text { Common Gateway Interface } \\ \text { CLI } & \text { Command Line Interface } \\ \text { CLR } & \text { Common Language Runtime } \\ \text { CMP } & \text { Container Managed Persistence } \\ \text { CPU } & \text { Central Processing Unit } \\ \text { DB } & \text { Database }\end{array}$

DBMS Database Management System

DBPRM Dynamic Bean Pool Resource Management

DNS Domain Name Service

EIS Enterprise Information System

EJB Enterprise Java Bean

ERP Enterprise Resource Planning

FIFO First In First Out

GIF Graphical Interchange Format

HTTP Hyper Text Transfer Protocol

HTTPS Hyper Test Transfer Protocol over SSL

I/O Input/Output

IAT Inter Arrival Time

IDL Interface Description Language 


\begin{tabular}{|c|c|}
\hline IIOP & Internet Inter-ORB Protocol \\
\hline J2EE & Java 2 Enterprise Edition \\
\hline J2SE & Java 2 Standard Edition \\
\hline JAF & Java Activation Framework \\
\hline JDBC & Java Database Connectivity \\
\hline JDK & Java Development Kit \\
\hline JMS & Java Messaging Service \\
\hline JNDI & Java Naming and Directory Interface \\
\hline JSP & Java Server Pages \\
\hline JTA & Java Transaction API \\
\hline JTS & Java Transaction Service \\
\hline JVM & Java Virtual Machine \\
\hline LAN & Local Area Network \\
\hline LDAP & Local Directory Access Protocol \\
\hline MDB & Message Driven Bean \\
\hline ODBC & Open Database Connectivity \\
\hline OMG & Object Management Group \\
\hline ORB & Object Request Broker \\
\hline $\mathrm{PC}$ & Personal Computer \\
\hline QoS & Quality of Service \\
\hline RAM & Random Access Memory \\
\hline RMI & Remote Method Invocation \\
\hline $\mathrm{RPC}$ & Remote Procedure Call \\
\hline
\end{tabular}


SMTP Simple Mail Transfer Protocol

SOAP Simple Object Access Protocol

SQL $\quad$ Structured Query Language

SSL Secure Socket Layer

URL Uniform Resource Locator

XML Extensible Markup Language 


\section{Chapter 1 Introduction}

\subsection{Distributed Systems}

The Internet is continuously growing in popularity and the number of servers and users around the world is increasing. The global trend is that location is of no importance, and that technology is the savior that will help overcome distance. Distributed systems is one such technology, which in recent years has become very important.

The characteristics of distributed systems are that they have concurrent components, they lack a global clock and their components may fail independently. Resource sharing is the main motivating factor for constructing a distributed system [8]. Resources can include Web pages or information dispersed through database records. Using the Internet, resources can be shared with users all over the world (i.e. the World Wide Web).

In a distributed system, different resources may also be in different locations. When multiple users access resources in different locations, the design of a distributed system must meet certain requirements, such as heterogeneity and concurrency. The design and development of distributed systems that consist of a large number of resources and have many users can become fairly complex, in order to cover all the requirements. To ease the complexity, a large number of technologies for the design and development of distributed systems exists. One is Enterprise JavaBeans (EJB), which is part of the Java 2 
platform Enterprise Edition (J2EE) technology. J2EE is a standard for developing multitier applications developed by Sun Microsystems.

\subsection{J2EE Overview}

J2EE provides an architectural framework for an enterprise application solution. It has the capabilities required to build multi-tiered e-business applications. The J2EE structure comprises three layers: the client tier, the Web tier, and the Business tier.

The J2EE Client tier is the end-user interaction platform. Included in this are Internet browsers, wireless devices, voice interfaces, Java applications, and Java applets. The primary invocation is via HTML over HTTP or HTTP Secure (HTTPS) for thin clients, and Remote Method Invocation (RMI)/Internet Inter-ORB Protocol (IIOP) for Java clients. As for the J2EE Web tier, it provides services that includes Web-based (HTTP) file access (e.g., HTML, GIFs, etc.), and Web programming interfaces (e.g. JSPs and Servlets). Finally, the J2EE Business Logic tier is the one that positions J2EE as an application server architecture rather than a Web programming architecture. Through the Enterprise JavaBean (EJB) framework, new business application components can be developed and assembled into Java Enterprise applications. Although available to the Web tier through RMI-IIOP interfaces, these EJBs are now accessible for non-Web processing applications as well. For instance, they could be used for application-toapplication interactions via Java Message Service messaging.

\subsubsection{EJB Overview}


J2EE provides different types of components for different purposes. Enterprise JavaBeans (EJBs) is one of the principal types of components in J2EE. In a typical J2EE application, EJBs contain the application's business logic and live business data. Although it is possible to use standard Java objects to contain business logic and business data, using EJBs addresses many of the problems that might occur when simple Java objects are used, such as scalability, life cycle management, and state management [24].

An EJB is essentially a managed component that is created, controlled, and destroyed by the J2EE container in which it lives. This control allows the container to manage the number of EJBs currently in existence and the resources they are using, such as memory and database connections [24]. Each container will maintain a pool of EJB instances that are ready to be assigned to a client. When a client no longer needs an EJB, the EJB instance will be returned to the pool and all of its resources will be released. At times of heavy load, even EJB instances that are still in use by clients may be returned to the pool so they can service other clients. When the original client makes another request of its EJB, the container will reconstitute the original EJB instance to service the request [30]. This pooling and recycling of EJB instances means that a few EJB instances, and the resources they use, can be shared among many clients. This improves the scalability of the EJB-based systems.

The client that uses the EJB instance does not need to know all the operations performed by the container. As far as the client is concerned, it is talking to a remote component that supports defined business methods. How those methods are implemented and any operation performed by the container, such as just-in-time instantiation of that specific 
component instance, are entirely transparent to the client part of the application [26].

The EJB benefits from certain services provided by the container, such as automatic security, automatic transactions, life cycle management, and so on. To do this, the EJB must conform to certain rules and implement an appropriate interface that allows the container to manage the component. The EJB is packaged with configuration information that indicates the component's requirements, such as transaction and security requirements. The container will then use this information to perform authentication and control transactions on behalf of the component-the component does not have to contain code to perform such tasks [26].

The primary purpose of the container is to control and provide services for the EJBs it contains [30]. When it needs to use some underlying functionality, such as creating a transaction on behalf of a bean, it uses the facilities of the underlying EJB server. The EJB server is the base set of services on top of which the container runs. Different types of EJBs will run in different containers, but many different EJB containers can run on a single EJB server. EJB servers are generally delivered as part of a J2EE-compliant application server (e.g. Borland Application Server [7], BEA Weblogic [32], JBOSS application server [15]).

\subsection{Motivation for the Thesis}

When considering Web-based businesses on the World Wide Web (e.g. e-Commerce), J2EE application servers continue to be one of the most popular server framework 
implementations in the industry today [5]. And since the World Wide Web community of users continues to grow in size, shape (i.e. different services offered online) and importance (i.e. day-to-day use of emails, messaging, etc.), the management of resources within an application server domain, in such a context, is of primary importance. Within a J2EE application server, Enterprise JavaBeans (EJBs) are the main central resources of an application server environment by which the business logic of choice is implemented. Once these resources are deployed and made available to a client(s) requesting a service, a J2EE framework offers a number of means for ensuring extendibility and load distribution over a clustered distributed environment. However, within a single J2EE server instance, available EJB resources are limited by the actual server hardware resources (i.e. processing power and memory), as well as by the pool size for deployed EJBs. Depending on the EJB type, the pool size for an EJB usually represents the minimum and maximum number of instances that an EJB's container would be managing, and is typically static ${ }^{1}$, once it has been configured and deployed.

This thesis explores an approach for dynamically managing the deployed EJB pool size configuration at run time, to make it more adaptive to the system state as captured by the number of requests currently active on the system. The thesis will first propose a new mechanism for Dynamic Bean Pool Management, and then compare the performance of current static pool configuration of EJB with the prototype implementation of the Dynamic Bean Pool Management.

\footnotetext{
${ }^{1}$ Once a bean has been deployed, its specified pool size configuration (i.e. minimum and maximum) remains unchanged at run time.
} 


\subsection{Goals of the Thesis}

The primary objectives of this thesis are to devise, implement and investigate a newly proposed dynamic bean pool management mechanism, and study its performance characteristics within a Web-based environment. The study focuses on comparing the new mechanism's performance with the performance of traditional static bean pool size configuration, on a single application server. This mechanism uses the Façade Patternbased stateless-session bean approach, as the access façade mechanism for deployed application server resources (given the stateless nature of most Web-based client/server interactions). It focuses mainly on dynamically managing the deployed stateless-session façades' bean pool resources by allowing its pool sizes to be adaptive, at run time, to the number of client's requests. This is accomplished through dynamically controlling the deployed stateless-session façades' bean pool sizes.

\subsection{Thesis Outline}

This thesis is composed of five chapters. Chapter 2 reviews the background of and challenges with distributed systems, and examples of its programming models, leading to J2EE architecture, and how such architecture addresses the outlined distributed systems' challenges. Chapter 2 also discusses the challenge of EJB pool size configuration management for improving system performance. Chapter 3 presents the Dynamic Bean Pool Resource Management technique proposed in this thesis. The experiments that were run, and analyses of the results gathered, are presented in Chapter 4. Chapter 5 provides conclusions and recommendations for future research. 


\section{Chapter 2 Background}

Existing work related to the research is presented. More general distributed system concepts are presented first, followed by related work in the J2EE area.

\subsection{Distributed Systems}

The importance of distributed system architecture has increased in recent years. Object Oriented-based knowledge and experience, along with the continuous evolution of such technology-based architecture, has led to the creation of distributed systems. Resource sharing was the main driving force behind the evolution of distributed systems [31][8], and Web pages, files, and database records are all examples of such shared resources.

The desire to improve performance led to the concept of client/server architecture. Appropriately, some of the processing was done on the client side, with the rest done on the server side for improved performance, as a result of reusing processing power that exists on both sides of the client/server architecture. Growing popularity of client/server

architecture in the business environment was presented in [8]. As such the number of tiers can differ depending on the architecture of choice and suitability. Unlike the traditional two-tier architecture, the client/server model, the three-tier model is continuing to increase in popularity within large distributed system environments. Three-tier architecture allows for the following functional separations in its architecture:

- Presentation tier:

This is the client's tier, which is mainly responsible for handling the presentation of the retrieved client's data (e.g. display rendering). 
- Logic (middle) tier:

This is where business rules and programming logic details are functionally defined and run.

- Data tier:

This contains the system's business data (e.g. database server).

\subsubsection{Challenges in Distributed Systems}

Depending on the characteristics of the distributed system, the following main challenges must be considered:

- Heterogeneity [31]

A distributed system can be composed of a number of different programming languages, computer system hardware, operating system, and networks. The Internet's communication protocols hide the differences between the networks, while middleware addresses other differences, such as data types that could be represented in various ways on different types of computer hardware.

- Openness [31]

Extendibility and ease of re-implementation are distributed system characteristics that allow for its openness. CORBA, for example, is published through a series of technical documents; the complete specification of the interfaces of its services is also published [20]. Systems that are designed to support resource-sharing in this way are termed "open distributed systems." This is mainly attributed to CORBA's architecture openness in publishing interfaces between communicating 
components, while facilitating an integration space between components (programs) written by different programmers.

A further benefit of open systems is that they are often vendor-independent.

- Scalability

A distributed system is described as scalable if it will remain effective when there is a significant increase in the number of resources and the numbers of users [31]. A good example of a distributed system that has witnessed an increase in its resources and users is the Internet, in which the hosts have increased drastically over time. Figure 1 shows the increase in the number of Web hosts with time.

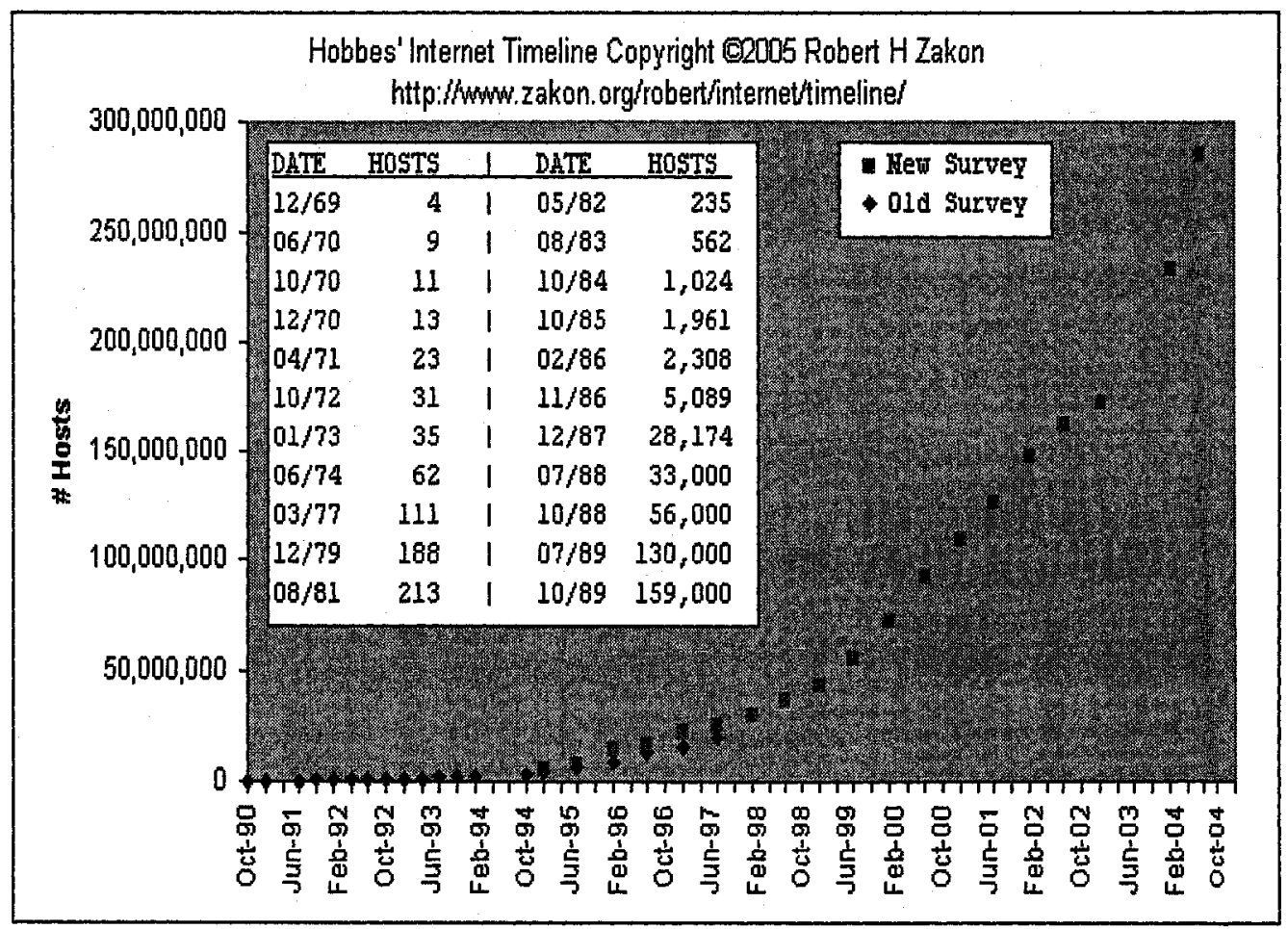

Figure 1: Number of hosts on the Internet [35]

- Handling Failure [31] 
In distributed systems, some components may fail while others continue to function. For this reason, each component needs to be designed to deal with those failures.

\section{- Concurrency [31]}

A distributed system provides its clients with sharable resources via its services and applications. Therefore, some clients may try to access a shared resource simultaneously. For example, a data structure that records bids for an auction can experience a heavy load due to frequent, and even simultaneous, access requests from its clients when a deadline nears.

Protection must be provided so that a client does not overwrite another client's data. Moreover, since requests can be in conflict with each other and produce inconsistent results, the rule in this case is that any object that represents a shared resource in a distributed system must be responsible for ensuring that it operates correctly in a concurrent environment. This applies to servers as much as to objects in applications. Thus, a programmer who takes an implementation of an object that was not intended for a distributed system must re-implement the object to be suitable for a safe, concurrent environment. In order to accomplish such a task, the operations of the newly incorporated object must be synchronized so that its data remains consistent. Synchronization can be carried out using certain standard techniques, such as semaphores [8].

- Transparency

Transparency allows certain aspects of distribution to be invisible to the application programmer so his/her attention is directed towards the design of the 
application instead. The programmer does not need to be concemed with unnecessary aspects like the location or the details of how other components access the application of interest. Thus, even network and process failures can be presented to application programmers in the form of exceptions that need to be handled. Access transparency and location transparency have been identified as being of key importance in addressing transparency [31], as those two transparency types tend to have a direct impact on the utilization of distributed resources.

\subsection{Programming Models for Distributed Systems}

One of the earliest programming models extended to facilitate the distributed programming context was the Remote Procedure Call (RPC) model. RPC allows client programs to call procedures on (remote) server programs running in different processes and on computer hardware that is separate from the client [31].

Remote Method Invocation (RMI) is used for objects in different processes to communicate with each other [31]. RMI is an extension of local method invocation; it allows an object that resides in one process to invoke the methods of an object residing in another process. Three RMI architectures that utilize these new techniques dominate today's market. A consortium called the Object Management Group, OMG, manages an architecture named CORBA, which stands for Common Object Request Broker Architecture. There is also Java RMI, which is SUN-developed RMI architecture. The 
third RMI-based architecture is Enterprise JavaBean (EJB). EJB is part of the SUN J2EE platform architecture, which will be described in further detail in Section 2.3.

The event-based programming model is yet another programming model whose applicability has been extended to distributed programs. The function of the event-based programming model is to allow objects to receive notification of the events at other objects in which they have registered interest [31]. This model has been extended to allow distributed event-based programs to be written.

\subsubsection{Examples of Existing Techniques of Distributed Applications}

\section{$\underline{\text { Java RMI }}$}

SUN developed Java RMI solely in Java, along with RMI's remote method and remote interface. Java RMI extends the Java Object model to support distributed objects in the Java language. In addition, Java RMI has no need for a special language for data type definition, as it is based on the Java Virtual Machine. Hence, objects can invoke methods on remote objects using a syntax that is the same as that of local invocations, not to mention that type checking applies to remote invocations and local invocations on an equal basis [31].

As a way of gaining transparency in Java RMI, remote method invocation and local method invocation use the same syntax, and hence reduce the difference between the two methods. However, remote invocations are more likely to fail than local ones, since they involve a network, another computer and another process. Disregarding which invocation 
semantic to choose, there is always the risk that no result will be received. In addition, in the case of failure, distinguishing between a failure of the network and of the remote server process is somewhat difficult. Nevertheless, objects making remote invocations must be able to recover from such situations. A point that may be of use here is that remote objects are distinguishable because they implement the Remote interface and throw RemoteExceptions [31].

\section{$\underline{\text { CORBA }}$}

CORBA is a middleware standard that facilitates communication between different applications. The communication between these programs is independent of their programming languages, their hardware and software platforms, and the network over which they communicate. CORBA provides mechanisms for remote objects location and invocation. One of the main purposes of establishing CORBA was to eliminate the programming language barrier between communicating applications, and hence implementers have the freedom to develop programs in any language. The applications that communicate through CORBA can communicate through an interface language, which is independent of any programming language.

CORBA is considered a multi-language RMI system. Thus, CORBA uses RMI as the remote method, and programmers need to learn the Interface Definition Language (IDL) as a result [31]. While learning IDL, the programmer should understand how the $\mathbb{D L}$ maps onto the implementation language. The programmer creates the IDL file, which 
upon compilation causes the generation of the stub and skeleton needed to create the ORB connection on both server and client sides [20] (see Figure 2).

The Object Request Broker, ORB, has been one of OMG's most noteworthy achievements, because it provides the user with a transparent method to invoke a method on an object. The role of the ORB in invoking a method involves locating the object, activating the object, and then establishing communication to the object for the client to send the request, whether the object is on the same machine or across the network. As far as transparency is concerned, when a client cannot communicate with a remote object, a remote invocation in CORBA throws an exception [31].

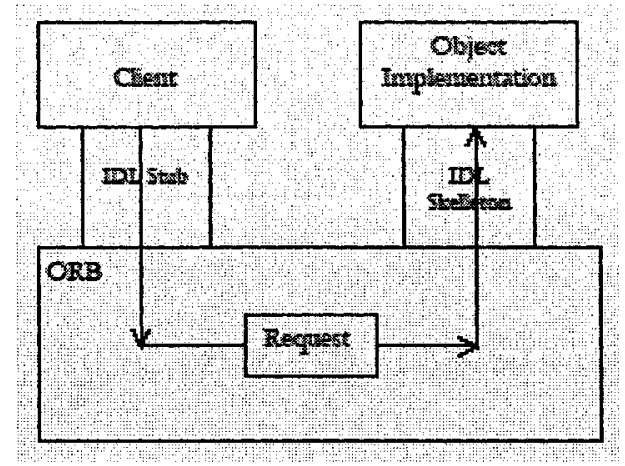

Figure 2: A request passing from a client to an object implementation [20]

\section{$\underline{M S . N E T}$}

.NET is Microsoft's platform for Extensible Markup Language (XML) Web services. XML Web services provide communication and data-sharing between applications over the Internet. Web services eliminate the dependence on an operating system or a programming language. The .NET framework consists of several parts, such as the Common Language Runtime (CLR). CLR is a rich set of class libraries for building XML 
Web services and Active Server Page (ASP) .NET. CLR manages the execution of code and provides services that make the development process easier. Any application that is implemented in .NET must have CLR as the essential component. CLR plays an important role in allowing components and applications designed in .NET to cross the language barrier, which enables these applications to integrate tightly with applications of different languages. In addition, CLR provides automatic memory management for selfdescribing objects, as well as the ability to compile once and run on any CPU and operating system that supports the runtime. Due to self-describing objects, IDLs are of no used in .NET applications [21].

A Web Service is a unit of application logic providing data and services to other applications. In order for applications to access Web Services, they use ubiquitous Web protocols and data formats like Hyper Text Transfer Protocol (HTTP), XML, and Simple Object Access Protocol (SOAP). Therefore, these applications do not need to worry about how each Web Service is implemented. Web Services gather the best aspects of component-based development and the Web, and these characteristics constitute a cornerstone of the Microsoft .NET programming model [21].

SOAP is a protocol specification for invoking methods on servers, services, components and objects [27]. SOAP is a lightweight and simple XML-based protocol. It allows for the exchange of structured and typed information on the Web, based on common open Web-based framework architecture. SOAP can support a number of mechanisms, including Remote Procedure Call (RPC), and it integrates well with a number of the existing Internet protocols (e.g. HTTP, Simple Mail Transfer Protocol (SMTP)) [27]. 


\subsection{J2EE Architecture}

Continuous cost increase in client-server software creation, deployment, and maintenance led to the emergence of the J2EE framework technology by Sun Microsystems. J2EE gained visible attention, as interest in the Internet and Web applications continued to grow rapidly, given higher demands for more complex and distributed Web-based service offerings [26].

The abbreviation J2EE stands for Java 2 Platform Enterprise Edition. J2EE is a platform created by Sun Microsystems Inc. to provide a Java-centric platform-independent environment for the online development, building, and deployment of Web-based applications. In doing so, J2EE provides platform independence, multi-user capability, and security within an enterprise class platform suitable for server-side, Java-based deployments. EJB, which is now a standard for building Java components for server-side applications, constitutes the main part of J2EE [26].

In order to understand the relationship between EJB and J2EE, as well as the reasons for developing the J2EE specifications, a description of the timeline of Java development evolution leading to the J2EE middleware specification is necessary. After introducing Java, Sun Microsystems focused on introducing a robust JDK (Java Development Kit), which then became a de facto implementation reference for Java. With the success of JDK and the recognized power of Java, the need for support for the server-side middleware was also recognized; this was a feature that Sun had not worked on developing up to this point. As a result, Sun Microsystems started to develop a set of 
enterprise-level APIs to provide adequate level services to support server-side deployments. The types of services provided by the enterprise APIs included transaction and lookup services, naming services, and version 1.0 of EJB APIs.

Application servers began to emerge in the market as a result of the new APIs a few months after the finalization of the EJB 1.0; the first of these was BEA's Weblogic [32]. These servers utilized the services newly introduced by the new Enterprise APIs. With this adoption of new services and APIs, new problems were identified and started to emerge. One of the problems was the evolution of the APIs themselves, as the APIs were simply seen as specifications for individual services, which resulted in each separate API evolving individually, with its own version number. As a result of the various versions of various APIs, and without a default reference implementation provided by Sun for the Enterprise APIs, compatibility issues arose. These problems, among others, triggered Sun Microsystems to develop a new robust middleware services suite to solve these problems and target server-side development. This suite was called the Java 2 Enterprise Edition (J2EE) [24][26].

Similar to previous generation APIs, J2EE continues to be a specification, not a product. These specifications define the rules for implementing and deploying J2EE compliant products and services. Figure 3 shows an example of how the technologies included with J2EE may be used. In this example, a client (e.g. web browser) makes an HTTP request to a web server for a web-page containing dynamic content that is generated by either a Servlet or a JSP. In turn, the Servlet or JSP may either connect directly to a database 
using JDBC, or may make a call to an EJB in a remote EJB server using RMI, to collect required data for its client's HTTP response. The EJB in this example would also use JDBC to collect data from the database.

Further details on technologies included in J2EE are described next.

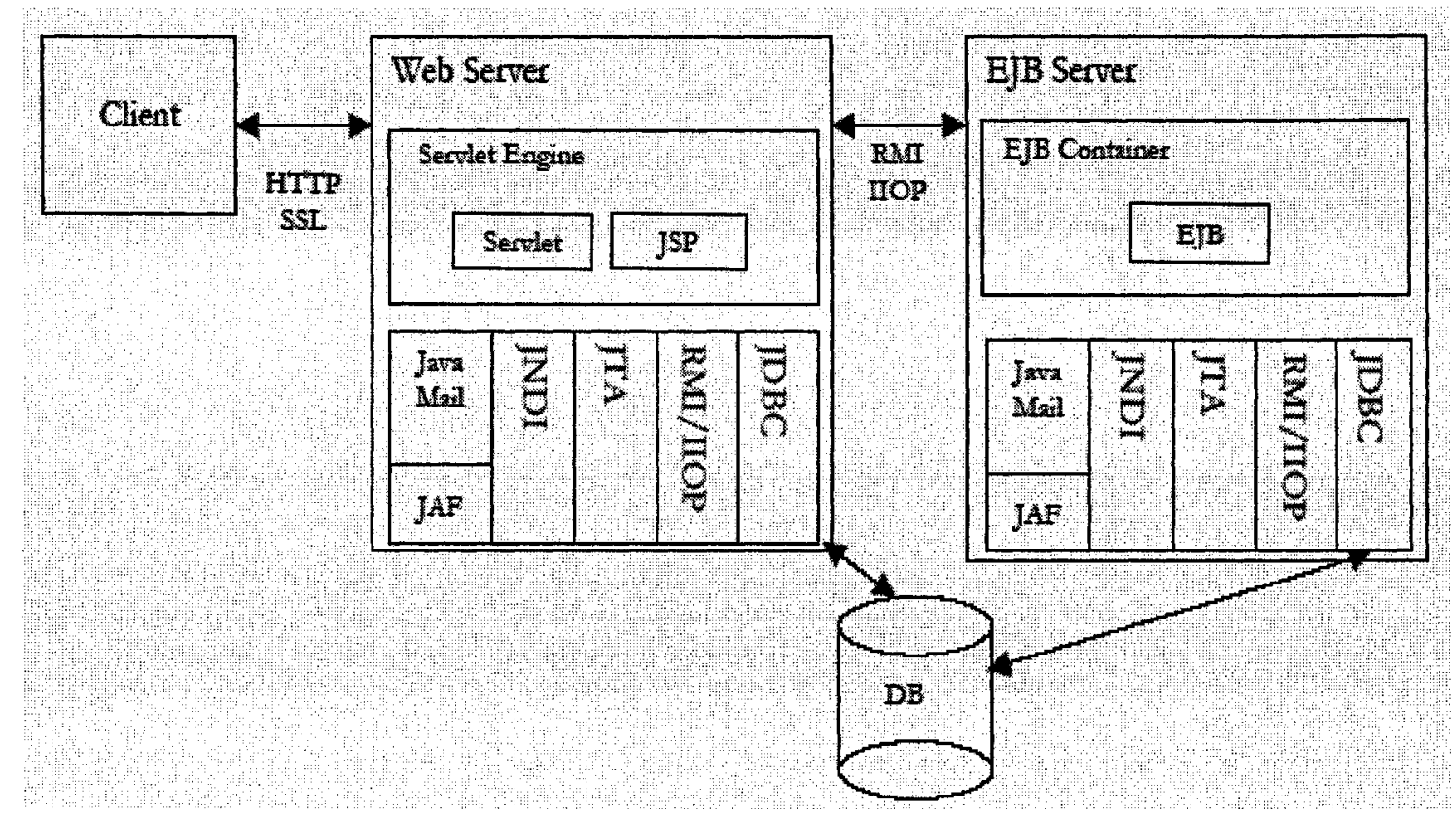

Figure 3: Example of a Web-based J2EE application [26]

Enterprise JavaBeans (EJB) - EJBs define the development and deployment specifications of how server-side components are written. EJBs also provide a standard interface between application servers and components utilizing standard contracts. EJBs will be discussed in further detail in Section 2.3.2.1.

Java Remote Method Invocation (RMI) and RMI-IIOP - RMI is a mechanism that allows the remote invocation of methods on other machines. Unlike traditional RPCs 
(Remote Procedure Calls), RMI abstracts such networking details as byte ordering and marshalling parameters away from the user. Another, more portable version of RMI is RMIIIOP, which uses the Internet Inter-ORB protocol (IIOP) developed for CORBA integration. IIOP provides a more robust protocol, which results in a better quality of service than that natively supported by RMI alone.

Java Naming and Directory Interface (JNDI) - JNDI is a naming and directory services standard that locates components and resources across the network. Other types of directory services, like Novell Directory Services (NDS) and Internet Lightweight Directory Access Protocol (LDAP), do exist, but they are in essence competing standards, each with different methods of access. JNDI provides a common standard access to different directory services by abstracting code so that it is no longer specific to a certain service. As a result, JNDI allows users to plug in and utilize various types of directory services without the need for code changes.

Java Database Connectivity (JDBC) - JDBC provides a bridge for relational database access. JDBC is described in more detail in Section 2.3.3.1.

Java Transaction API (JTA) and Java Transaction Service (JTS) - JTA is a highlevel interface used by applications to control transactions. A transaction is by definition a unit of work that makes guarantees about its execution, allowing different users to modify the same data while keeping each modification isolated. JTS, on the other hand, is the underlying set of low-level interfaces that are used by EJBs to facilitate 
transactions, without the need for user interaction or the need to handle such low-level details. JTS is based on CORBA's Object Transaction Services (OTS), which allows for collaboration among multiple vendors' transactions in heterogeneous distributed networks.

Java Messaging Service (JMS) - JMS provides reliable asynchronous message-passing between distributed objects. Asynchronous messaging between objects allows for increased system scalability, as processes are able to respond to messages when they are available.

Java Servlets and Java Server Pages (JSPs) - JSPs and Servlets represent a set of networked components that are intended for request-/response-based computations, making them ideal for HTTP client interactions.

Java Interface Definition Language (IDL) - IDL allows for the possibility of using CORBA objects within Java. It also utilizes OMG IDL or IIOP to allow for integration with other languages.

JavaMail - JavaMail service allows for platform and protocol independent e-mail messaging from within Java applications.

Java Activation Framework (JAF) - JAF is the framework for determining the type of data and encapsulating access to it. 
It should be noted that J2EE builds on Java 2 Standard Edition (J2SE) with the capabilities necessary for providing a secure, stable, fast, and complete platform for enterprise-level implementations. This also means that a J2EE-compliant application implements $\mathrm{J} 2 \mathrm{SE}$ as a subset of $\mathrm{J} 2 \mathrm{EE}$.

Figure 4 illustrates J2EEs' three tiers (based on the J2EE application presented in Figure 3). Figure 4 describes the construction of a traditional application using EJB as the middleware. These tiers will be described in further detail in the following sub-sections.

\subsubsection{Presentation Tier}

This top tier is responsible for the client interface. In a client/server environment, it can either reside entirely on the client side, or be split between the client and the server. The J2EE components that belong to this tier are discussed next.

\subsubsection{Servlets}

Servlets are typically a form of Java applets that reside on Web servers rather than on Web browsers. The main purpose of Servlets is to provide execution functionality to generate dynamic content, as well as secure access to Web-based data. Since their introduction, Servlets have become a popular alternative to the Common Gateway Interface (CGI) method of code execution. However, unlike CGI, Servlets are persistent within the server environment, allowing them to serve multiple requests without the 


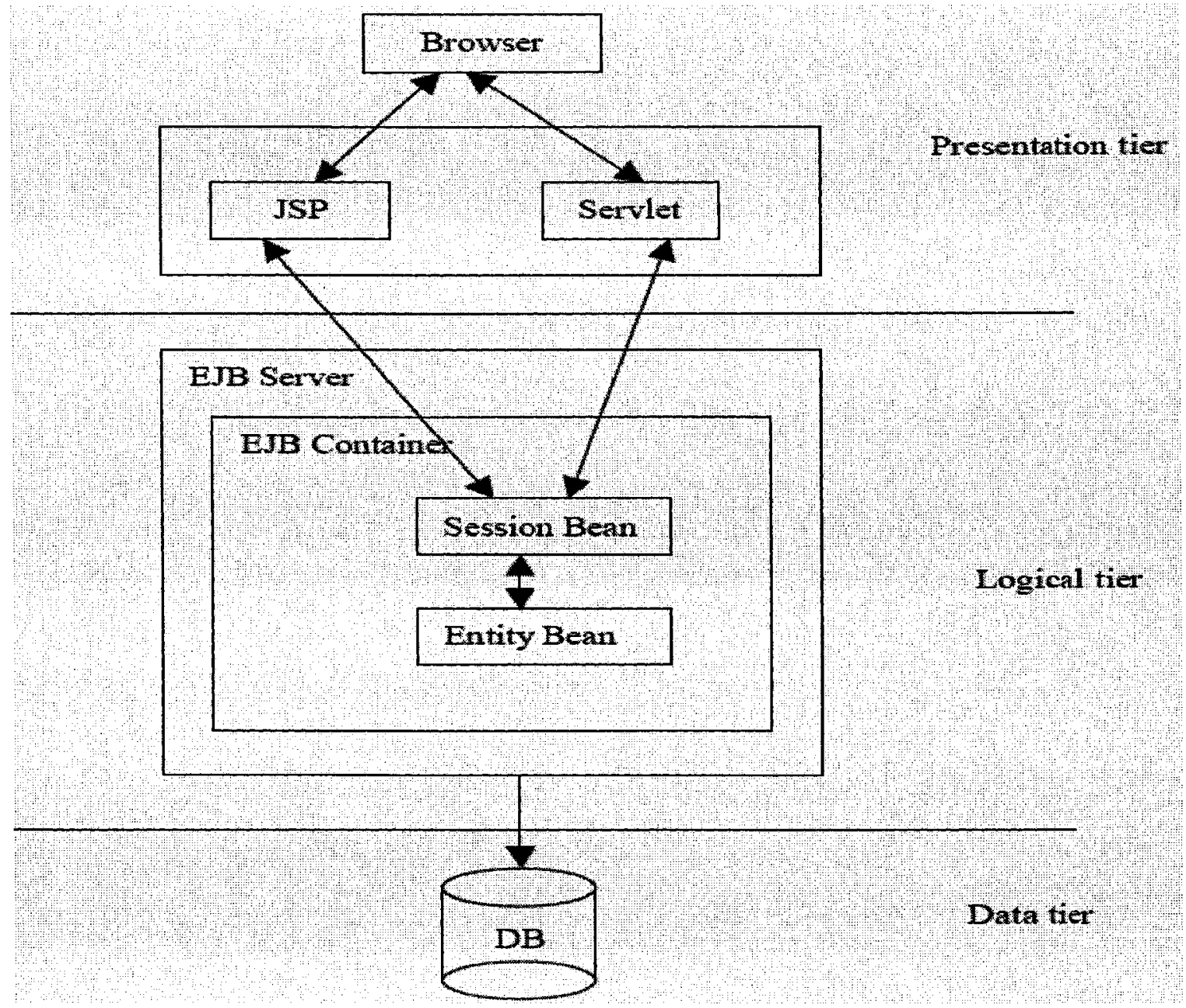

Figure 4: Example of a three-tier architecture with EJB as middleware

wasted time of starting the process, setting up the environment, and post-execution cleanup, as is the case with CGI. In addition, because Servlets are Java based, they are platform independent, and can leverage the Java libraries and database access methods for HTTP calls.

Servlets require a runtime environment to execute and be hosted on the server. This runtime environment is, in essence, a container for the Servlets, and can be either a specifically designed application server or any general type of container capable of 
providing the needed functionality: a Java-enabled Web server, for example [24]. This needed functionality includes the ability to decode requests and format responses, as well as provide the network services required for the Servlets to communicate. Needless to say, support for the HTTP protocol is a basic one needed for the Servlets containers, although additional protocols that enable request-response style interactions may also be supported [24].

\subsubsection{JSP}

JSP is a server-side extension that allows for building dynamic Web pages by combining Java with HTML. JSP was created by Sun Microsystems to provide functionality similar to Microsoft's Active Server Pages (ASPs). JSP can be seen, in simple terms, as HTML pages that contain executable code that allows for the application logic to create dynamic content. In doing so, JDBC, EJB, Java Beans, and/or RMI objects can be used, and since JSP is Java and HTML based, it is inherently platform-independent [28].

\subsubsection{CORBA Clients}

J2EE does not require its clients to be either Web-based or Java-based in order to access J2EE-based server applications. J2EE provides support for CORBA clients that can be written in any language. This support comes from J2EE's use of RMI/IDL in the EJB distributed object model. CORBA clients are out of the scope of this thesis and so will not be discussed here. 


\subsubsection{Logical Tier}

The logical tier is typically split between both the client and the server. The functionality, however, can be split either way: the majority can either be on the server side (when it is referred to as a fat server), or on the client side (referred to as a fat client).

\subsubsection{EJB}

Before the introduction of J2EE, multi-tier implementations used a variety of different standards, as discussed previously. These standards caused various limitations, including lack of flexibility in building and deploying standardized component-based applications, as well as the lack of scalability to meet business needs. In addition, these standards resulted in stressing the focus on building a compatible mix of operating systems, platforms, and middleware so that they could function properly. EJBs (Enterprise JavaBeans) were designed to overcome these problems [24]. EJB is part of the J2EE specification and provides a Java API, which is capable of acting as middleware. EJBs are more complex than regular Java Beans and are Java-written server components that are distributed using RMI. Similar to Servlets, EJBs require a container within which they can execute and are restricted from certain types of operations, mainly for security purposes. Restrictions include concurrent threads, and listening on sockets [24][30]. The concurrency restriction, however, is mainly due to the coordination of access to resources; in doing so, EJBs queue the requests and allow for resource access, one request at a time [26]. This, however, resulted in the recognition of a need for parallel processing abilities within EJBs. This need led to the emergence of Message-Driven Beans (MDBs). MDBs will be described in Section 2.3.2.1. 


\section{Application Server}

The term "application server" typically refers to software that hosts and offers various services. A J2EE Application Server is intended to offer a runtime environment for server-side components and allows the use of off-the-shelf middleware components and services [24]. As a result, J2EE and EJB allow for the deployment of independently developed compliant components on almost all Java application servers. In doing so, J2EE specifications split the application server into the following:

1) EJB Container - The EJB container hosts the EJBs and provides the environment for them to execute and be able to provide services. Any given container can host many EJBs. In addition, it also provides interface visibility to the client side [24]

2) EJB Server - The EJB server can be seen as a container for the EJB containers. It provides services like security, naming, and transaction handling to the beans via interaction with the EJB container that hosts them. The containers herein act on behalf of the beans to mediate services offered by the EJB server. Unfortunately, J2EE does not define the interface between the EJB containers and servers, eliminating the possibility for development of general purpose interacting containers and servers. For this reason, companies provide EJB application servers with both the container and the server part within the same server package. 


\section{Enterprise Bean Components}

EJB applications consist of a number of Enterprise JavaBeans; each bean in turn consists of a set of components. These components can be developed or provided by the tools within the EJB container to allow the bean to handle both data and business logic. Figure 5 illustrates these components and is described further.

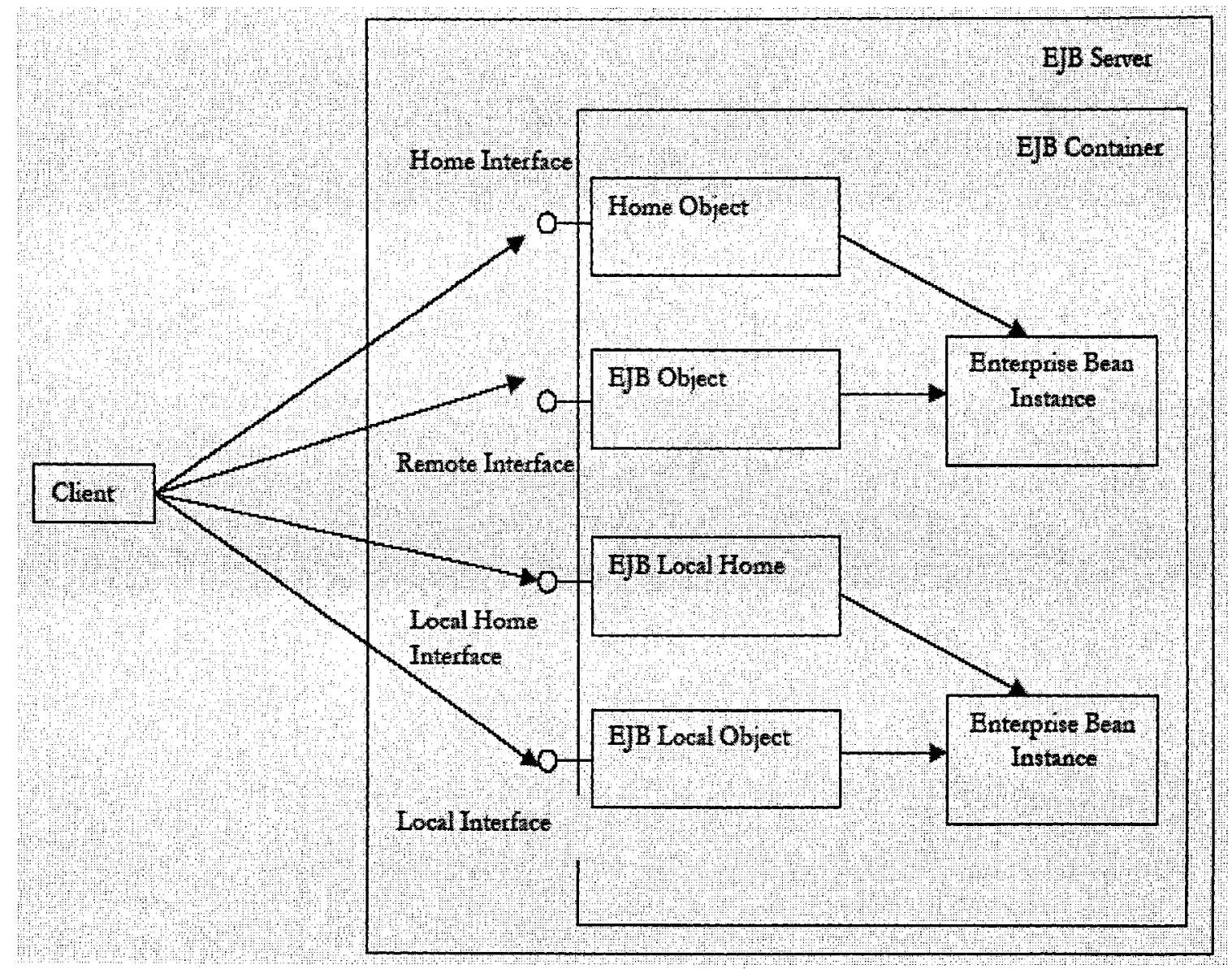

Figure 5: The enterprise bean components [30]

Bean class - The bean class is responsible for implementing the logic in the bean and, as a result, contains most of the EJB application code. The Bean class is encapsulated inside 
the bean and can only be accessed by the client via the bean's local and remote interfaces. In addition, a context interface that allows information to be obtained from the containers is also provided with Enterprise JavaBeans.

Home interface - The home interface is responsible for defining methods a client uses for locating, creating, and destroying enterprise bean instances.

Remote interface - The remote interface is responsible for defining the bean's business methods, which can later be accessed by the client via the remote interface. A client uses the remote interfaces to access these methods.

Local interface and local home interface - The local interface provides lightweight, direct interaction among beans that exist in the same JVM, eliminating the need to use remote method calls and eliminating the overheads associated with them.

EJB object and EJB local object - Provided by the EJB container; implements both the local and remote interfaces between local enterprise beans.

EJB home object and EJB local home object - Provided by the EJB container; implements both the local home and home interfaces. Both objects are responsible for managing the lifecycle-related operations within the bean instance; the local home object, however, is provided particularly for use among local beans. 
Figure 6 illustrates the way in which a business method would be accessed in an enterprise bean. The client starts by looking up the home interface of the bean using JNDI. JNDI returns the home object reference, which can then be used to request creation of a new instance of the remote interface, from the EJB container. The EJB container creates a new EJB object, and gets a reference to it back to the client.

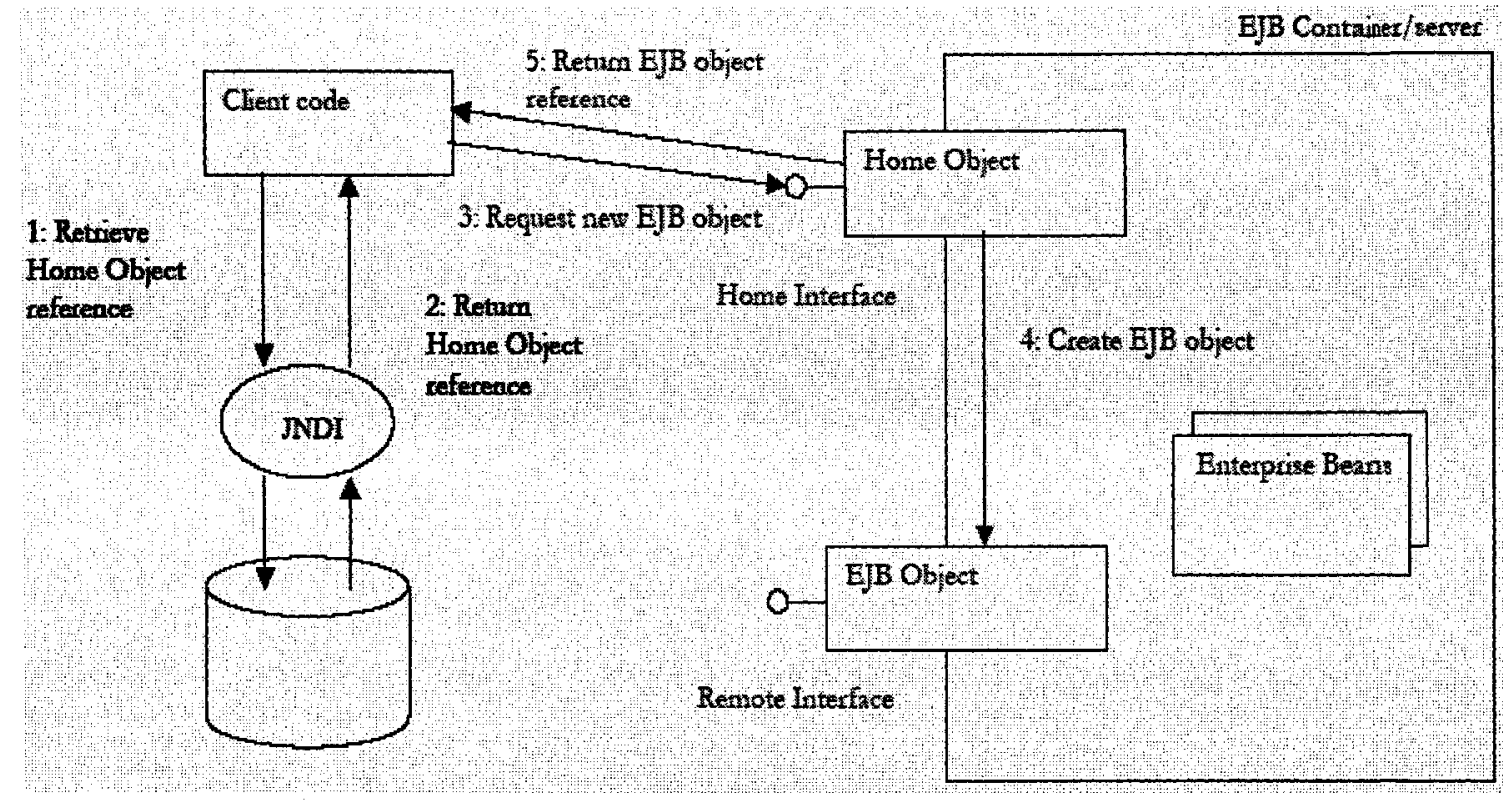

Figure 6: Retrieval of the home interface and generation of a remote interface instance [30]

Figure 7 describes the final steps that follow the steps depicted in Figure 6, for the way in which a business method would be accessed in an enterprise bean. With the reference of the EJB interface now returned to the client, the client is able to call the EJB object's methods, which then handles the calls to a bean instance in its EJB container, and gets the returned result back to the client. When a client calls a method on an EJB remote interface, its EJB container would seek to acquire a bean instance and delegates the method to acquired bean instance. Once the bean establishes its called method's result, the result value gets in turn sent back to the client. 


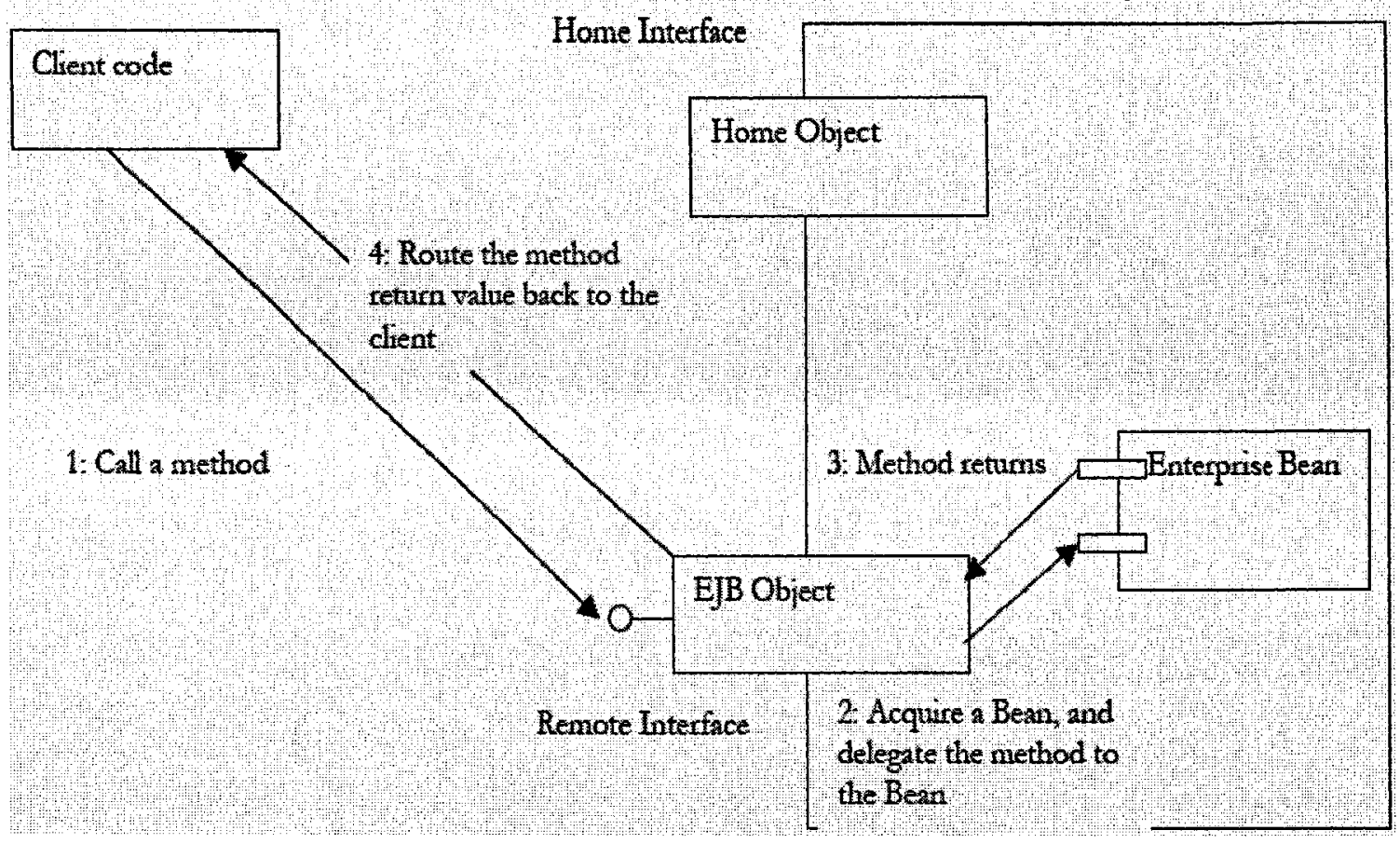

Figure 7: Handling a client request to a business method [30]

Deployment descriptor - Describes the method of deployment for each bean in the EJB application and the way they are handled by the container. The deployment descriptors are a must for EJB applications, and a minimum of one descriptor needs to be defined per application. Application servers, however, typically require more deployment descriptors to accommodate the server's specific settings [29]. In the case of EJBs deployment descriptors, and depending on the type of bean being deployed, the deployment descriptor includes a specification for either pool size configuration (i.e. Stateless Session, Message Driven, and Entity EJBs), or cache size configuration (i.e. Stateful Session, and Entity EJBs). Further details on EJBs are provided in Section 2.3.2.1. Pool size configuration refers to the specification of minimum and maximum pool size that an EJB container is to manage, while cache size configuration usually refers to the maximum number of EJB 
objects that are allowed in the disk cache, where the managed state can be passivated ${ }^{2}$ in to $[26]$.

EJB specifications define three different types of beans [30]: message-driven beans, session beans, and entity beans. Each bean type is described.

\section{$\underline{\text { Session Beans }}$}

A session bean is a bean tied to a given session between one client and an application, responsible for representing this client to the application. Session beans terminate when the session between the client and application terminates. Session beans are of twotypes:

1) Stateful: where the state of the object is maintained on behalf of the client. A stateful session bean acts as a server-side extension of the client that uses it. It gets created by a client and will work for only that client until the client connection is dropped or the bean is explicitly removed [26]. An example of this is a shopping cart application, in which the session bean keeps track of the selected items and can be used to calculate the total cost.

2) Stateless: the session bean does not keep a state on behalf of the client, allowing a given instance of the bean to serve more than one client by not terminating when the client terminates its session [26]. Conceptually, the business methods on a stateless session bean are similar to procedural applications or static methods; there is no instance state, so all the data needed to execute the method is provided by the method arguments.

\footnotetext{
${ }^{2}$ Passivation is the process by which a J2EE application server removes an EJB instance from cache while preserving its state on disk. While passivated, EJBs are not in memory and are not immediately available for client requests, as they are when in the cache $[32,4]$.
} 


\section{Entity Beans}

An entity bean represents an object in some persistent storage. Persistence is the process of coordinating the data represented by a bean instance with the database [26]. The persistence of an entity bean can either be managed by the EJB container, or by the entity bean itself. If the data storage is a relational database, every entity bean represents one table in the database, and every instance of the entity bean represents one record of that table.

Advantages of using entity beans instead of accessing the database directly are listed [26]:

- Objectification of data provides a simpler mechanism for accessing and changing data. SQL commands are abstracted.

- Software reuse is also possible, since once an entity bean has been written, it can be used for the entire project.

- It is possible to define entity properties according to the business concepts rather than the underlying data formats, which means that the business purpose can be clearly defined and the data format can evolve separately.

There are two types of entity beans: container-managed persistence (CMP) entity beans and bean-managed persistence (BMP) entity beans. The most important difference is that the container is responsible for the persistence for the CMP bean. The BMP bean, on the other hand, transfers all responsibilities to the developer. 


\section{$\underline{\text { Message-Driven Beans }}$}

Message-driven beans are responsible for processing asynchronous messages for J2E applications and were first included in version 2.0 of the EJB specifications. Message driven beans have neither remote nor home interfaces, unlike entity and session beans. They are currently only capable of processing JMS messages, although this may change in future specifications [24] [30].

\section{$\underline{\text { Transaction Management }}$}

The transaction service is an essential component of the EJB architecture and removes development complexity from the bean provider and application developer. EJB does not support nested transactions. The EJB container implements the necessary low-level transaction protocols, such as the two-phase commit protocol, transaction context propagation and distributed two-phase commit protocol. It must also provide support for the JTA API [30].

The EJB transaction service consists of three types of services: bean-managed, clientmanaged and container-managed. Bean and client managed transactions work the same way, and it is the developer's responsibility to get them to work. These transaction types are useful when more control over the transactions is needed. One restriction of beanmanaged transactions is that only session and message-driven beans can use them. When container-managed transactions are used, the container is responsible for providing the 
transaction delimitation for the enterprise beans as it was specified in the deployment descriptor during deployment [30].

\subsubsection{Data Tier}

The data tier is the lowest tier in a three-tier application, and consists of some kind of persistent storage. The data tier is located on the server side in a client/server system. The most common kind of data in a J2EE solution today is a relational database. Of course, the data tier may consist of many other types of data; for example, a file system or a legacy ${ }^{3}$ system. An important feature of new software products is the ability to work with a company's legacy applications, or at least be able to import data from them. The data tier may also consist of other kinds of databases; for example, an object-relational database and an object database. The connection between the logical tier and the data tier can be made in two ways: using JDBC or using connectors.

\subsubsection{JDBC}

JDBC is a Java API that enables Java programs to execute SQL statements. JDBC can be seen as the connection between the logical tier and the data tier. This allows Java programs to interact with any SQL-compliant database. Since nearly all relational database management systems (DBMSs) support SQL, and because Java itself runs on most platforms, JDBC makes it possible to write a single database application that can

\footnotetext{
${ }^{3}$ In information technology, legacy applications are those that have been inherited from languages, platforms, and techniques that are older than the current technology [33].
} 
run on different platforms and interact with different DBMSs. JDBC is similar to ODBC (Open Database Connectivity), but is designed specifically for Java programs, whereas ODBC is language-independent. JDBC was developed by JavaSoft, a subsidiary of Sun Microsystems [33].

\subsubsection{Connectors}

Connectors are a part of the J2EE specification [29] and manage integration with existing Enterprise Information Systems (EIS). The J2EE Connector architecture defines a standard architecture for connecting the J2EE platform to heterogeneous EISs. Examples of EISs include $\mathrm{ERP}^{4}$, mainframe transaction processing, database systems and legacy applications not written in the Java programming language. By defining a set of scalable, secure and transactional mechanisms, the J2EE Connector architecture enables the integration of EISs with application servers and enterprise applications.

The J2EE Connector architecture enables an EIS vendor to provide a standard resource adapter for its EIS. The resource adapter plugs into an application server, providing connectivity between the EIS, the application server, and the enterprise application. If an application server vendor has extended its system to support the J2EE Connector architecture, it is assured of seamless connectivity to multiple EISs. An EIS vendor needs to provide just one standard resource adapter, which has the capability to plug into any application server that supports the J2EE Connector architecture.

\footnotetext{
${ }^{4}$ ERP is short for enterprise resource planning, a business management system that integrates all facets of the business, including planning, manufacturing, sales, and marketing [33].
} 


\subsubsection{How has EJB Addressed Distributed Systems Challenges?}

The challenges of developing a distributed application are described in Section 2.1.1. This section evaluates how EJB has addressed these challenges.

\subsubsection{Heterogeneity}

An EJB system can only be written in the Java programming language. Nevertheless, the client of an enterprise bean can be written in a different programming language [30]. This is made possible through the Java IDL. EJB 2.0 requires EJB implementations to support RMI/IIOP [26].

A client view of an EJB is independent of the implementation of the EJB and its container [30]. Theoretically this ensures that a client application is portable across all container implementations in which the entity bean might be deployed. In real life, one major portability problem depends on how the developer implements the EJB system. Most of the vendors that implement application servers and containers also implement a couple of extra features not stated in the EJB specification. Applications using these features cannot be moved directly to another vendor's application server without modification. Although moving one's application to another application server is not very common, but the ability to handle heterogeneity is decreased regardless.

\subsubsection{Openness}

The entire Enterprise JavaBeans specification [30] is available on the Internet. Any 
vendor may implement an application server and a container that supports the specification.

\subsubsection{Scalability}

The EJB container is where all enterprise beans are executed. A client's name space can be configured to include home interfaces from multiple containers.

These are used for load-balancing and clustering, which give more stable and scalable systems. One of the advantages with EJB systems is that they can handle a substantial load and at the same time maintain good performance [24]. Two mechanisms help scalability: instance pooling and passivation/activation. Instance pooling is an old technique that is traditionally used to offer faster database connections. By letting business objects share connections, the number of connections is decreased [24].

\subsubsection{Failure Handling}

Application's exceptions thrown by an enterprise bean's implementation are usually handled by the developer. As to internal EJB system level exceptions, EJB specification recommends propagation of internally raised system exceptions (e.g. by the bean's container), for appropriate handling by the enterprise developer [30].

Another way in which EJB handles failures is that data in a database can be recreated from an entity bean if the database fails and, in the same way, the entity beans can be recreated from the database tables if the application server fails. Session beans cannot be recreated in case of failure. 


\subsubsection{Concurrency}

Session beans do not support concurrency [24][30]. Clients must therefore create their own instances of a session bean or let different EJB objects share instances from the instance pool. Consequently, session beans avoid the concurrency problem for distributed systems by not allowing concurrency. Many clients can be connected to the same EJB object, but only one client at a time is allowed to use the bean. EJB container handles the fact that only one client at a time can be connected to the same EJB object.

Entity beans do support concurrency by allowing several entity bean instances to access the same underlying data. However, concurrency is not supported by the EJB specification with respect to clients accessing entity beans. As in the case of session beans, several clients are not allowed to access the same instance of the bean concurrently, and the container handles that.

EJB specification does not support nested transactions. Because of this, EJB cannot have the same functionality of a middleware application that uses SQL for database transactions.

\subsubsection{Transparency}

A client accesses a session bean or an entity bean through the bean's remote or local interfaces. These interfaces of the bean provide the client view. The container provides 
classes that implement the enterprise bean's interfaces. Although classes implemented by the container provide the client view of the deployed enterprise bean, the container itself is transparent to the client [30]. The client of a session bean or an entity bean may be a remote client or a local client, depending on whether the client makes use of the enterprise bean's remote or local client view interface. The local client view is new in the EJB 2.0 specification.

The remote client view of an enterprise bean is location-independent [30]. A client running in the same JVM as a bean instance uses the same API to access the bean as a client running in a different JVM on the same or different machine. Further, EJB specification allowed the client view of a session object to be independent of the implementation of the session bean and the container [30].

\subsubsection{Configuration challenge for improving enterprise system performance}

\subsubsection{System configuration challenge of Enterprise applications}

Enterprise applications present a management challenge on many fronts, the most obvious being their sheer abundance. With Web-based enterprise information technology striving to handle the demands of many developed and packaged applications, it is easy to see why managing their performance is often a reactive, problem-driven task. On the other hand, developed applications tend to be more passively managed according to a traditional life cycle model, as depicted in Figure 8. Once applications are deployed, it is more difficult and costly to make changes to its operating context (i.e. required hardware 
deployment and software configurations). The emphasis therefore moves to incremental functional improvement and maintenance, as opposed to proactive optimization and performance management.

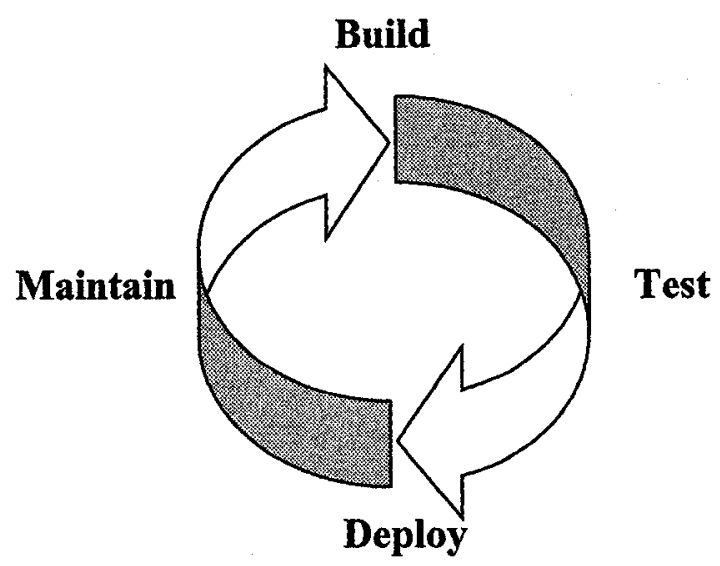

Figure 8: Management of enterprise applications according to a common life cycle [16]

Rising complexity intensifies the challenge of optimizing application performance. Often, Web-based applications' broad functionality and scale require a tiered architecture that, when implemented, involves multiple layers of servers and communications components (as discussed in Chapter 2). Thus, in such an environment, the interaction of Web-based applications and clients accessing such applications across the network is unpredictable. Since the workloads of e-business sites, which obviously rely on an underlying Webbased applications infrastructure, tend to fluctuate dynamically and exhibit short-term variations [18], even if the system is configured properly at a given point of time it will show poor performance at other times. That is, a statically configured system will perform poorly in a dynamic workload environment such as the e-commerce 
environment. As such, optimizing system configuration becomes a challenge for these kinds of (Web-based) enterprise applications.

\subsubsection{Addressing the software configuration challenge}

A number of researchers have worked on addressing the software configuration challenge. However, it must be noted that software configuration is divided into two levels: the application level and the system level (or infrastructure level). On the application level software configuration front, the efforts of [1][10] mainly addressed mechanisms required for achieving application level software configuration. Component redundancy was applied in [10] to enable J2EE applications to dynamically select the most appropriate components in each particular execution environment, in order to optimize the performance of the application as a whole.

Feedback control architecture to deliver different Web content to Web applications according to the server CPU utilization, in order to ensure the performance of Web applications, was presented in [1].

However, in J2EE middleware, its application configuration is not as important as its system configuration, in which performance is of utmost importance in building distributed systems. Failure to meet the performance requirements can lead to failure of the entire system.

System level configuration was addressed in [25][19]. The effort of [25] identified a feedforwarding control mechanism to allow for predictive autonomic systems. Experiments in 
[25] show worse response times due to incorrectly forecasting control measures for overload parameters in some periods. Therefore, it is not enough to only use a feedforward control approach in a dynamic configuration situation. On the other hand, the effort in [19] used hill-climbing techniques, combined with analytic queuing models, to guide the search for the optimal number of server threads to attain desired QoS levels. Since J2EE application servers are complex systems, the approach in [19] requires detailed information on the system to construct queuing models, which is hard to obtain in complex systems such as J2EE application servers. Further, within dynamic configuration environments, it is not an easy task to build a queuing model that takes transient effects into account. The research presented in this thesis is different from the approaches outlined in these papers, and uses simple indicators of system state to adaptively control key configuration parameters of an application server.

There has been little work on adaptive self-configuration for J2EE application servers. Research presented in [23] tries to find good application server configurations using an empirical approach. However, they only provide a rule of thumb for manually configuring application servers. The framework proposed by [10] focuses on J2EE applications, not on J2EE application servers, and their application adaptation is still performed by an admin managing the application. The research presented in [17] provides an approach to predict the performance of EJB component-based applications during the design phase of software development. The approach constructs a quantitative performance driven design model for a proposed application. Results gathered from the design model are used to choose from different application architectures. Two different 
EJB application architectures were implemented and measured in [17] to validate their approach. However, much like [23] and [10], the research effort in [17] does not address the issue of adapting the application's system configuration to clients' dynamic behavior, at run-time.

With J2EE-based middleware architecture, the need to address its application server (resources) configuration in an adaptive manner is becoming apparent [34][22][12]. This research focuses on adaptive configuration of $\mathrm{J} 2 \mathrm{EE}$ application servers that is discussed in details in Chapter 3.

\subsubsection{J2EE application server configuration}

J2EE-based middleware systems reduce the complexity of building large distributed systems by hiding the low-level networking details from the programmer and providing ready-to-use services such as transactions and security for applications. However, the complexity of J2EE-based middleware systems introduces new challenges to system configurations [22].

Performance is a key to many software systems. Appropriate configuration of software systems is necessary to meet desired performance.

The performance of a J2EE application server depends on the configuration of the deployed EJB components services [22]. The EJB component model isolates applications and beans, for the most part, from the details of the component services configuration parameters that control the EJB's resource deployment environment. Such parameters 
control EJB's transaction nature, persistence, resource pooling, and security management $[30]$.

For good performance, these parameters of a J2EE application server have to be configured manually, due to a lack of adaptive self-configuration capability. However, this kind of manual configuration is time-consuming and requires highly skilled personnel [26]. Thus, it poses the challenge of fostering a self-adaptive configuration mechanism for these kinds of enterprise application server middleware systems [12]. This research focuses particularly on EJB resource pool configuration, to achieve high system performance.

\subsubsection{The EJB pool configuration challenge}

As part of the many J2EE application server configuration control parameters, deployed EJB resource pooling is of interest in this thesis. EJB pool size configuration control is of relevance to stateless session beans, entity beans, and message-driven beans, excluding stateful session beans. This is mainly due to the fact that stateful session beans are not intended to be used by more than a single client at a time [26]. As such, a stateful session bean can only be created for a specific client, where it maintains the managed clients' state during the course of its existence [26][30]. 
Pool sizes are generally provisioned via the respective containers' minimum and maximum pool size parameters, which are specified in the deployment descriptor of an EJB. Where:

Minimum pool size - the number of bean instances the container maintains in the pool as a minimum, irrespective of how long the beans have been in the pool.

Maximum pool size - the number of bean instances the container maintains as a maximum. This ensures there is an upper limit to the number of pooled beans, so memory and server CPU usage do not grow in an unbounded fashion. [26]

The creation and destruction of beans is expensive [24][26][9]. To reduce this cost, the EJB Container/Server creates a pool of beans that depends on a vendor-specific configuration; one needs to give a proper value to this pool size to increase performance. For example, Weblogic's weblogic-ejb-jar.xml has an element <pool> [32], and JBoss server's jboss.xml has an element <instance-pool> [15]. These elements are used to allow the specification of pool size configuration.

The maximum number of beans in a pool impacts performance. If this is lower, then the Container has to put the clients in the queue when the number of clients accessing it is more than the maximum pool size. This degrades performance and client results take more time to complete. Thus, specifying proper pool size configuration becomes very important, and a challenge on its own, especially when taking into account the unpredictability of Web-based clients accessing such server resources in real-time. 


\section{Chapter 3 Dynamic Bean Pool Resource Management (DBPRM)}

The importance of pool size configuration within an unpredictable Web-based client access environment was discussed in Section 2.3.5. This thesis presents a newly implemented server-side technique that improves J2EE system performance for an enterprise Web-based application. This technique monitors the number of client requests within a controlled client access tier. The designed access tier is based on a stateless session EJBs façade ${ }^{5}$. The proposed technique controls the pool size of the stateless session façade (access tier) at run-time, to adapt to the number of client requests. The technique is called Dynamic Bean Pool Resource Management (DBPRM), and it operates based on pre-configured usability thresholds and reconfiguration factors for the controlled stateless session façade EJBs.

\subsection{DBPRM Solution Architecture}

The DBPRM solution is intended to address J2EE system application performance for Web-based applications. As such, its solution architecture is composed of the following two tiers (see Figure 9):

- Web-Tier: This tier is based on a Servlet implementation of an enterprise Web application and therefore contains the server-side Web application Servlet. This Servlet is directly dependent on using the DBPRM mechanism's main objects, which it imports as its EJB access utility. The DBPRM mechanism deployed

\footnotetext{
${ }^{5}$ Based on adopted Session Façade pattern [3]
} 
within this Web-tier controls access of the Servlet's clients to a designed enterprise stateless session façade tier. Section 3.3 provides further details on the designed functionality of the DBPRM mechanism.

- Stateless Session Façade Tier: Given that the majority of the World Wide Web traffic is stateless in nature [35], this thesis was primarily concerned with addressing the performance enhancement of Internet client access that is stateless in nature. Thus the DBPRM architecture solution incorporates this stateless session façade tier (composed of stateless session EJBs) as the controlled enterprise application façade tier of its architecture. The embedded DBPRM mechanism within the Web-Tier of this architecture controls access to any stateless session bean within the façade tier. Section 3.2 presents further details on the adopted session façade pattern, and part of this tier's architecture.

\subsection{DBPRM's Adopted Session Façade Pattern}

The stateless session bean façade is designed to be the only enterprise application tier that provides access to any other resources beyond it (i.e. entity beans, message-driven beans, etc.) in our DBPRM architecture design solution. This follows the adopted (stateless) Session Façade pattern, which would control access to the EJBs' container, and their deployed beans, via the implemented DBPRM mechanism. Section 3.2.1 presents a definition of the Façade pattern, and the design strategy chosen from it is outlined (i.e. the stateless session façade strategy). 


\subsubsection{Definition of Session Façade Pattern}

The Session Façade pattern is a J2EE core design pattern for an Enterprise JavaBeanbased application design [3]. This pattern was based on the Façade Design pattern for Object-Oriented software [11]. The pattern allows for the use of a session bean as a façade to encapsulate the complexity of interactions between the business objects participating in a workflow. This Session Façade manages the business objects, and provides a uniform coarse-grained service access later to clients" [3].

In essence, the Session Façade is considered to be positioned as a business-tier controller layer that controls the interactions between the client and the participant's business data and business service objects (e.g. Session beans, Entity beans, Message-driven beans) [3].

In a complex application, there may be numerous Session Façades that can intermediate between the client and these objects. Where a Session Façade might be useful can be identified by studying the client requirements and interactions that are typically documented in use cases and scenarios.

The Session Façade pattern was intended to be used in either of the following two enterprise design strategies:

- Stateful Session Façade Strategy

"A business process that needs multiple method calls to complete the service is a conversational business process. The conversational state must be saved between 
each client method invocation. In this scenario, a stateful session bean may be a more suitable approach for implementing the Session Façade"' [3].

- Stateless Session Façade Strategy

"A business process that needs only one method call to complete the service is a non-conversational business process. Such processes are suitably implemented using a stateless session bean" [3]. This is the strategy of choice for DBPRM's session façade tier, as explained in Section 3.1 .

\subsection{DBPRM Mechanism}

DBPRM provides an enterprise Web application with a dynamic control mechanism for the pool size configuration of its stateless session façade's EJBs. Figure 9 illustrates the adopted design architecture flow of the DBPRM mechanism, as part of the design of this research.

The DBPRM mechanism is a utility class object used by the Servlet of our Web-Tier architecture (see Figure 9). Once the Servlet is deployed and running, it instantiates the DBPRM object, and registers within it all Stateless Session Beans (Façade's beans). It then relies completely on the DBPRM utility's mechanism to access EJBs in the façade. Figure 9 depicts the flow of the DBPRM utility within our Web application solution architecture, and in light of clients' requests.

The flow steps shown in Figure 9 are as follows: 


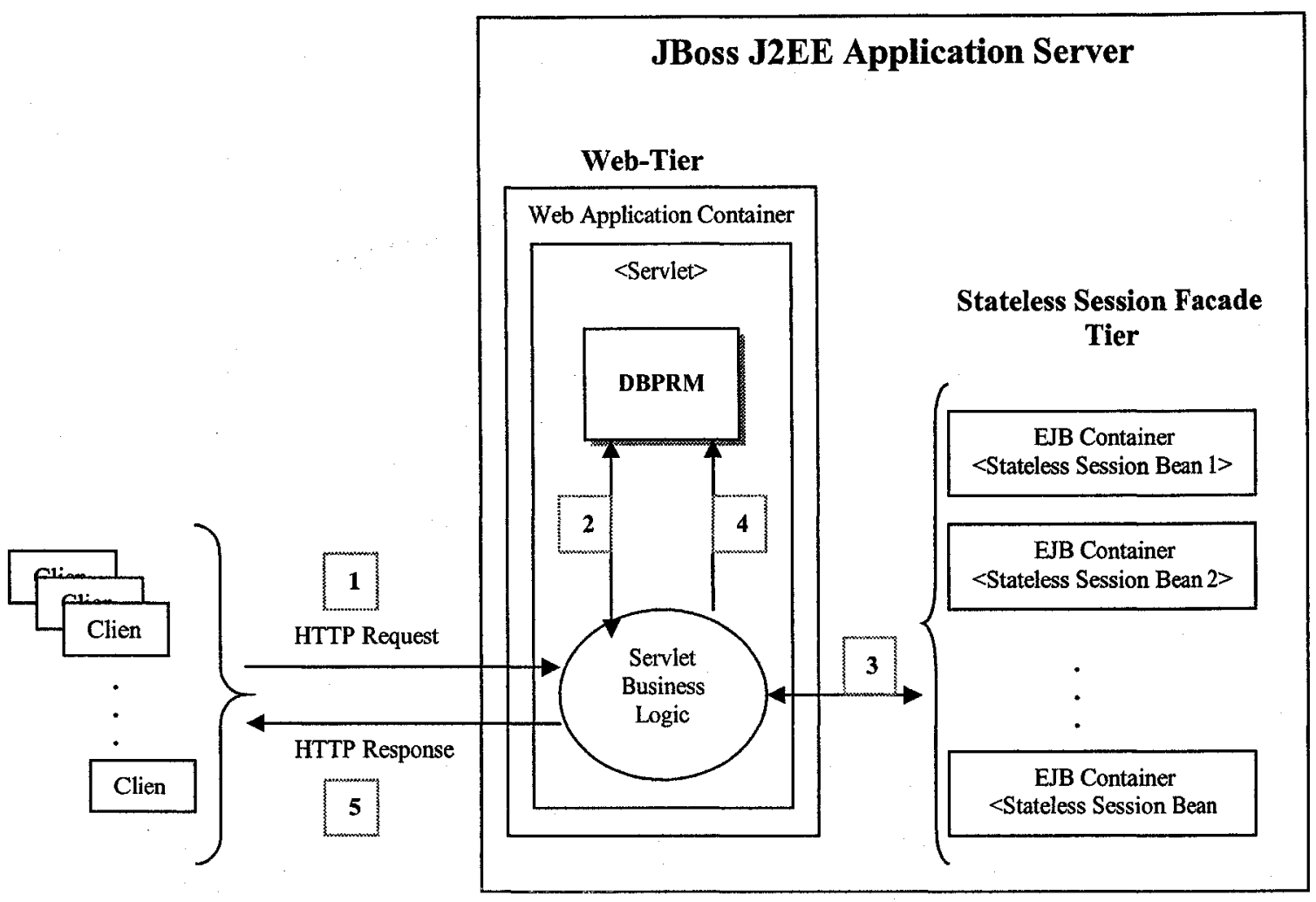

Figure 9: DBPRM operational architecture

[1] Clients send HTTP requests to the Servlet. Each request's URL includes a reference to the specific EJB container bean type (bean name) with which it wants to associate its request with. The reference would identify the façade's stateless session bean type that a client wishes to have access to. The HTTP request's URL also includes the service time for the façade's bean (i.e. CPU execution time in milliseconds). Section 4.1.1 provides details on the URL of the HTTP request issued by a client.

[2] Client's Servlet thread requests permission to get access to the façade's bean from its DBPRM utility, which monitors and controls client access to the registered 
stateless-session bean façade. If there are no available bean instances in the EJB container of the stateless session bean to which the client is requesting access to, the Servlet's DBPRM utility will seek to reconfigure its managed façade pool sizes to facilitate for one if possible (see Figure 10 for details). Otherwise, the DBPRM utility will queue the client request until one is available.

[3] Once the Servlet's client thread has received the requested reference of the stateless-session bean from its DBPRM utility, it then proceeds to call and run the required business logic from the stateless-session bean reference it acquired. In our solution prototype, this is done by calling a method on the stateless session bean, while passing to it the desired execution time, which forces the bean to run in a loop until the specified time has passed.

[4] When the Servlet's client thread has completed the call to the business logic of its acquired stateless session bean, it then notifies the DBPRM utility that it has completed use of its stateless-session bean. In turn, the DBPRM utility updates its monitored state for the returned bean's DBPRM pool size configuration (See Section 3.3.1 for details).

[5] Finally, the client's Servlet thread sends back a response to the requesting client.

It is important to note that Servlets are based on a threaded (asynchronous) framework, and each client request to a Servlet is handled via a separate thread of that Servlet [13]. All the Servlet's threads share the DBPRM utility object, which gets instantiated at the time of main Servlet deployment and creation in the Web server [13]. 


\subsubsection{DBPRM's Bean-Pool-Configuration and Algorithm}

Deployed façade's stateless-session beans are registered with the DBPRM utility at startup time. At this point it is important to recall the EJB deployment descriptor details presented in Section 2.3.2.1. At the time of deployment of the stateless session beans, the J2EE's application server deployment descriptors require the specification of deployed bean pool sizes (e.g. minimum and maximum pool sizes) [29]. Standard EJB deployment descriptor pool size specifications are necessary for the EJBs' container [29]. However, the DBPRM mechanism disregards the EJB container's pool size specification, and takes over the control of pool size. In order to disregard the container's pool size specification provided by the standard EJB deployment descriptor, our proposed solution prototype sets an open-ended maximum pool size for the EJBs. That is, the Max number of beans in pool is set very high for each deployed stateless session bean of our proposed solution's façade's EJBs. This is to allow for pool size configuration to be controlled at run-time by the DBPRM utility. As such, the set DBPRM pool size configuration of each stateless session bean registered with the DBPRM utility will be the effective pool size configuration.

Each Stateless session EJB of our proposed solution's façade has the following DBPRM configuration parameters specified for it, at the time of its registration with the DBPRM utility object. These parameters are controlled and monitored by the DBPRM mechanism (i.e. The DBPRM utility object): 
- Minimum pool size (PMin): This is the pool size that was initially specified for an EJB, when it was registered as part of the DBPRM façade's EJBs. DBPRM never attempts to reconfigure this value at run time, as DBPRM regards this parameter as the absolute minimum pool size configuration that will be sustained.

- Maximum pool size (PMax): This is the maximum pool size that was initially specified for an EJB when it was registered as part of the DBPRM façade's EJBs. It is important to note that DBPRM dynamically controls this parameter. As such, this parameter will be reset at run-time, by the DBPRM mechanism, to allow for DBPRM's dynamic adaptation of pool size to the number of client requests. Figure 10 presents the DBPRM mechanism's general algorithm, which provides details on how this parameter configuration is controlled.

- Pool over-use time threshold (OT): This is the threshold time, in milliseconds. If the time an EJB is waiting for an available bean exceeds OT, the DBPRM attempts to increase the pool size for this EJB, to allow for the required pool size adaptation to the number of client requests on this bean.

- Pool under-use time threshold (UT): This is the threshold time, in milliseconds. If the time for which an EJB has not received a request exceeds this threshold, then the DBPRM mechanism will be considering to reduce this EJB pool size to increase the pool size for other beans managed by the DBPRM mechanism. The DBPRM mechanism uses this parameter to determine the under-used EJBs in our proposed solution's façade. The DBPRM mechanism would then be able to recompute, at run time, the required decrease in pool size, for such under-used EJBs 
to increase the pool size of another EJB that encounters a number of client requests that is higher than its current pool size.

- Pool increase factor (IF): This is the factor by which a DBPRM mechanism increases a façade's EJB pool size. For example, If IF $=0.5$, then the increased pool size would be original-pool-size $\times 1.5$.

- Pool decrease factor (DF): This is the factor by which a DBPRM mechanism decreases a façade's EJB pool size. For example, If $D F=0.5$, then the decreased pool size would be original-pool-size $\times 0.5$.

- Activate DBPRM (AD): This is a boolean parameter that identifies whether or not a given façade's bean, would have the DBPRM's dynamic pool adaptation mechanism activated for changing its pool size. If set to false, for a given façade's EJB, then the DBPRM mechanism would only apply the standard pool size that was specified for the EJB (i.e. the EJB pool size would be bounded by only its set Minimum and Maximum pool size specifications).

It is important to note that the DBPRM mechanism performs the adaptation of façade's bean pools sizes, by reducing the EJB pool sizes in the façade that are least used (i.e. where their Under-use threshold has been exceeded the most). This is shown in line 8 to 15 of DBPRM's algorithm in Figure 10.

The DBPRM mechanism always keeps track of the current pool size for each registered EJB. The current pool size lies between the DBPRM-maintained Minimum (PMin) and Maximum (PMax) pool sizes. 
Figure 10 presents a high level algorithm for DBPRM's dynamic adaptation mechanism of EJB pool sizes, to (concurrent) client request rate. This algorithm shows how the DBPRM mechanism re-computes the façade's EJB pool sizes. This is done by first determining the desired increase in pool size, based on the increase factor (IF) of the EJB in need of the increase (Line 1 of Figure 10). Then, the DBPRM mechanism, searches the EJB pools to locate the least used EJB pool, for which the under-use threshold time has been exceeded the most (Line 8 to 15 of Figure 10). Once this leastused EJB pool is found (Line 18 of Figure 10), the DBPRM mechanism re-computes its pool size as follow:

- Check to see if the difference between the maximum pool size (PMax) and the current pool size (EJB-current-pool-size) of this least used EJB is sufficient for the desired pool increase (Line 20 to 23 of Figure 10). If not, then the DBPRM mechanism performs the next pool size re-computation steps (Line 24 of Figure 10):

- In Loop 2, DBPRM continues to decrease the least-used EJB's maximum pool size by its set decrease factor (DF), until the minimum pool size for this least-used EJB is reached (Line 27 to 39 of Figure 10).

- The DBPRM then checks to see if desired pool size increase value was reached. If not (Line 40 to 41 of Figure 10), the DBPRM mechanism looks for the next least-used EJB pool in the façade (if available), and repeats the same described pool size computation process (Line 7 to 42 of Figure 10). 


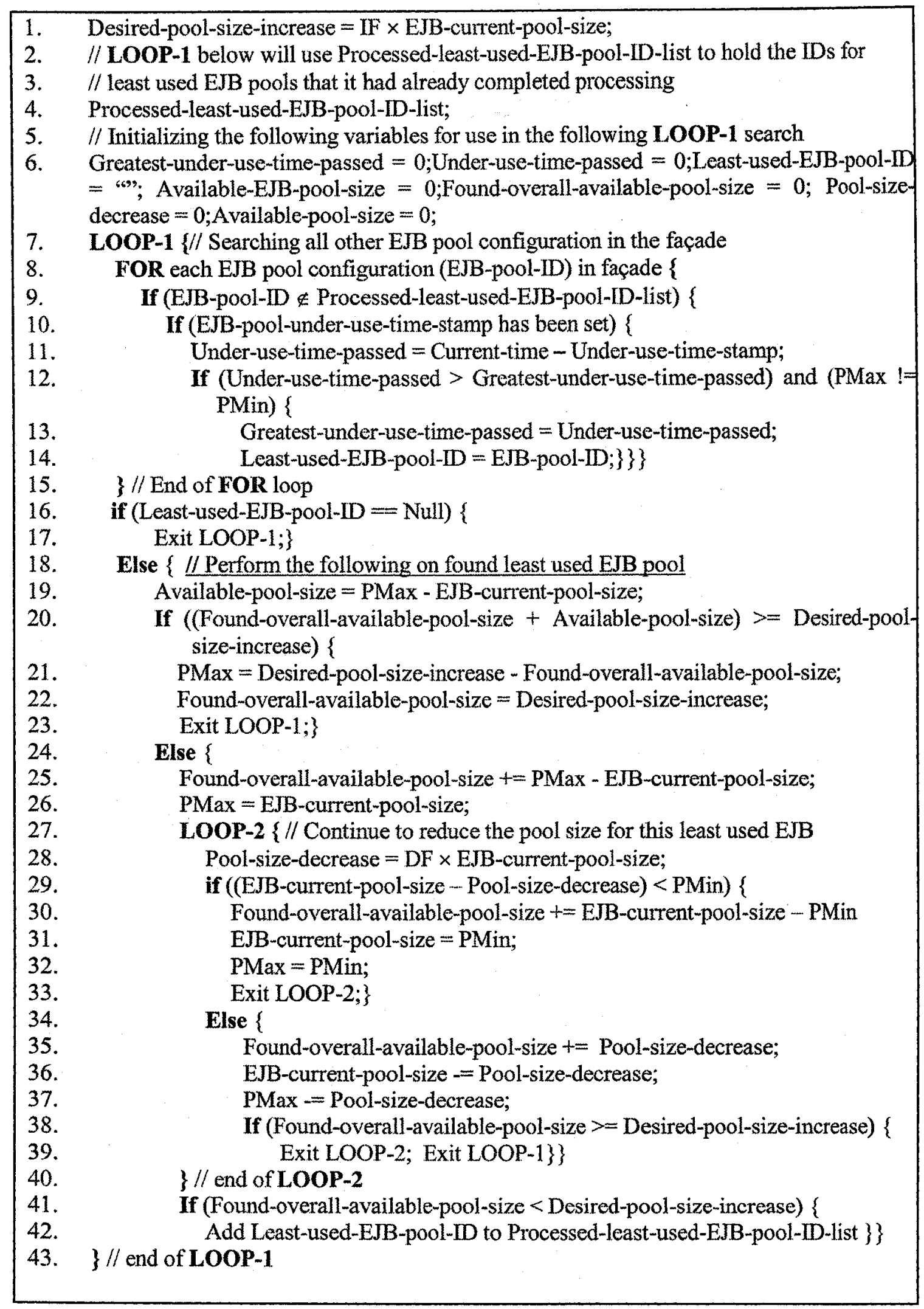

Figure 10: DBPRM Dynamic Adaptation Algorithm 


\section{Chapter 4 Experiments Conducted}

\subsection{Testing Environment}

The environment used to perform experiments for this research included two PCs on the same LAN. One PC was used as the server, with JBoss Application Server V3.0.8 installed on it (Pentium III $866 \mathrm{MHz}$ processor, with 256MB RAM, and Debian GNU/Linux 2.0 based operating system). The other was the client machine (Pentium III $500 \mathrm{MHz}$ processor, with 128MB RAM, and Windows ME operating system), running the Java-based Uniform Resource Locator (URL) Performance Analyzer tool. This tool was built entirely as part of this research effort. Section 4.1.1 provides further details.

\subsubsection{URL Performance Analyzer Tool}

This is a Java application that allows for stress testing a J2EE application server's Web engine, by generating client Hyper Text Transfer Protocol (HTTP) requests and measuring response time.

A number of important parameters that are tunable from the application's Command Line Interface (CLI) and URL data configuration file are presented:

- Total number of HTTP requests (RN)

The total number of client HTTP requests to be made on an HTTP server for the duration of the stress testing.

- Number of concurrent clients $(\mathrm{CN})$ 
This is the number of concurrent threads handling HTTP requests initiation and response time measurements. The application refers to those threads as "Foreground Threads". Each thread picks the URL of an HTTP request from a First in First out (FIFO) request queue (see Figure 11) managed by this application. The thread then sends the HTTP request, and computes the response time.

- Mean request inter-arrival time for client requests (IAT)

The performance analyzer application's main thread manages a queue of arrived client requests generated by another internal "Background Thread". This "Background Thread" generates an HTTP request and inserts it into the client request queue. The inter-arrival times are exponentially distributed, and the mean is given by this parameter.

- Service time (S)

This specifies the mean service time for the generated HTTP requests. Each HTTP request's URL address string carries the service time for which its EJB is expected to execute for. Note: EJB execution is not based on I/O operations. Rather it is based solely on CPU operation (i.e. continuous loop until the specified service time duration completes). The URL Performance Analyzer tool will specify the service time for each HTTP request, depending on the user requirements for service time distribution.

Figure 11 illustrates the architecture of this application: 


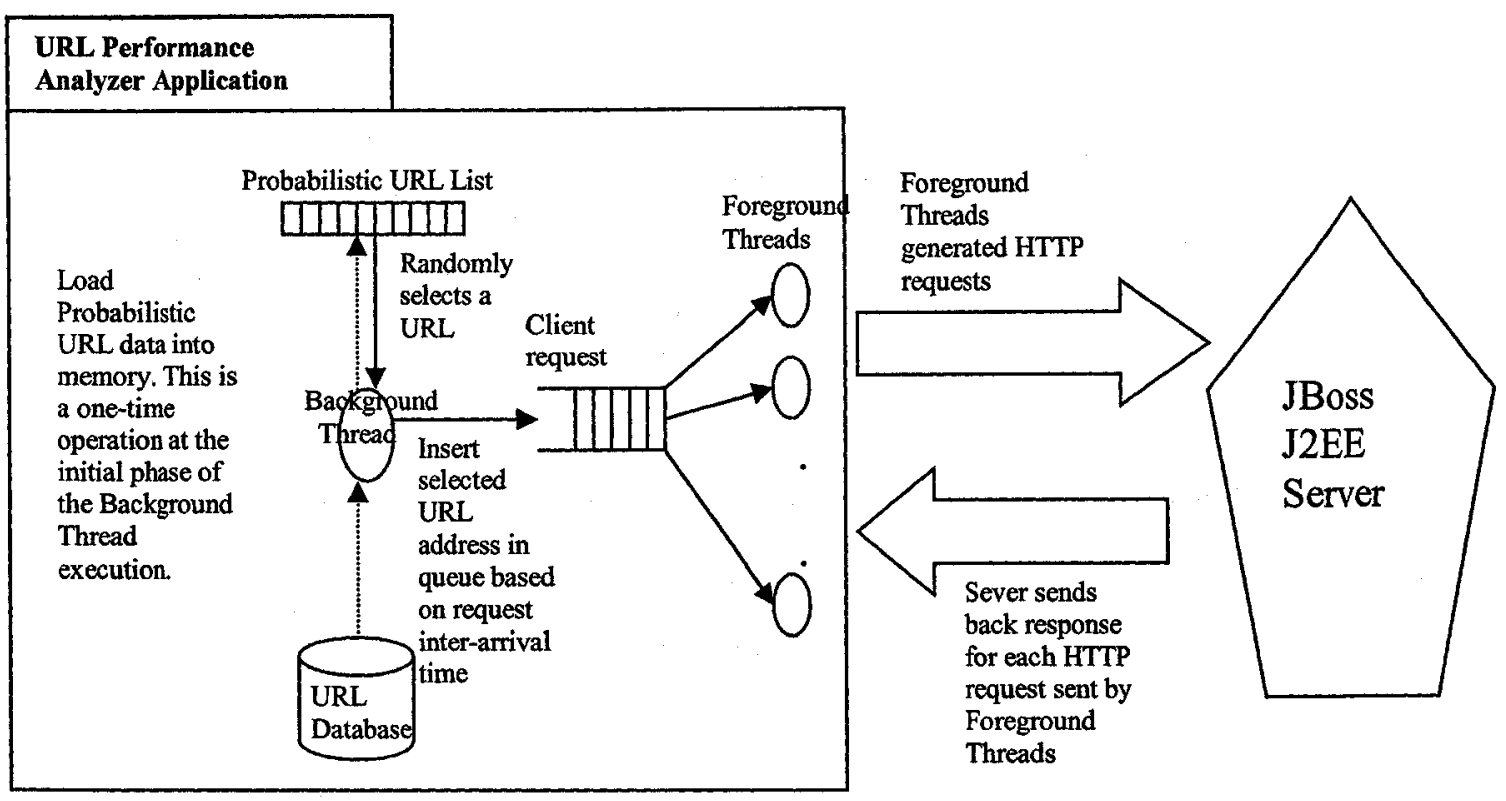

Figure 11: URL Performance Analyzer Design Architecture

The Background Thread in this tool randomly selects and removes an HTTP request address (URL) from its created URL list (array). Each request has a probability of occurrence that is an input parameter for this tool. The URL list gets created by the Background Thread during its initial phases of operation, based on the specified probability for each URL loaded from the program's URL database.

The proportion of the number of entries of a given URL in the list is equal to the probability of its occurrence. Once the URL list has been created, the Background Thread randomly selects and removes a URL from this list to insert into the application's client request queue. This is repeated until the total number of entries in the queue is equal to RN. 
The illustrated URL database in Figure 11 is based on a text file from which the URL Analyzer loads its HTTP client requests. This file contains line records; each line represents an HTTP client request's URL, along with the desired probability of such HTTP requests to be made during the course of a test run of the URL Performance Analyzer application. Each line record is as follows:

Probability $>$ URL

The following is an example of a line record from an HTTP client request's URL, with $70 \%$ probability of this URL request being generated. The example also shows that the URL will request a Servlet to call a specific EJB (EJB_A) from the stateless session façade (see Section 3.1), along with a specified EJB service time of [S] in milliseconds.

0.7>http://192.168.1.2:8080/Servlet?SessionFacade=EJB_A\&EJBServiceTime=[S]

The service time in this URL is the service time expected from the EJB for this request. URL Performance Analyzer tool specifies this service time for each request based on the user requirements. The tool will assign a fixed service time for each request, based on the specified service time. Or the tool can assign a service time value for each request, based on an exponential distribution with the specified mean service time.

The performance analyzer application uses this file for generation of HTTP requests. Appendix A shows further design details for the URL Performance Analyzer tool. 


\subsection{Results of Experiments}

This section presents the experiments that were performed, as well as an analysis of the results that were gathered. Before running these experiments, preliminary experiments were performed for determining the experiment run length and the number of initial observations to be removed for eliminating the effect of startup transients.

\subsubsection{Common Parameters for Performed Experiments}

The experiments performed in this chapter had the following configurations in common:

- The number of concurrent clients used in all experiments was 30 . This was mainly attributed to a limitation in the client-side CPU processing; the client machine that was used could not handle more than 30 clients (which resembled concurrent threads—see Section 4.1.1 for details).

- With the exception of the experiment described in Section 4.2 .2 , all other experiments had the following stateless session façade configuration. The façade was composed of three different deployed stateless session EJBs (Bean-A, BeanB, and Bean-C). All deployed EJBs had the same method for their client interface, which allowed for the specification of service-time (bean CPU execution-time) for each client request made to it. Each deployed EJB had its own container that managed the EJB's deployment descriptors' pool size configurations (Section 2.3.2.1). The deployment descriptor of each EJB in performed experiments had the following pool size configurations:

- Minimum pool size was set to 1 . This is the initial pool size configuration. 
- Maximum pool size was set to 10,000 . This was an exaggerated maximum pool size, set in the EJB's J2EE deployment descriptor. This was to allow the maximum pool size to be non-restrictive to the maximum pool size configuration managed by DBPRM. Further, considering that the total number of concurrent clients (i.e. the Performance Analyzer Applications' foreground threads) used in all experiments in this chapter was 30, the maximum desired pool size limit could potentially be up to only 30 as the effective maximum desired pool size.

Each EJB was also registered with DBPRM, with the following initial pool size configurations (See Section 3.3 for DBPRM's pool size parameters details):

- PMin $=1$, similar to the container's deployment descriptors' EJB minimum pool size specification.

- PMax could vary depending on experiments.

\subsubsection{The effect of pool size on client response time}

This experiment shows the effect of EJB pool size configuration on response time. One stateless session bean was deployed for this experiment (named BeanA). The method used for its interface allows the service-time (bean executiontime) to be specified for each client request made. The maximum pool size for the deployed stateless session EJB was varied in the experiment. The fixed parameters are listed. 


\section{Fixed parameters for the experiment:}

- Exponentially distributed Inter Arrival Time with a mean $=110$ milliseconds

- Bean-A related configuration:

○ $\mathrm{S}$ (fixed) $=100 \mathrm{~ms}$

- Probability of requests made against this bean $(\mathrm{P})=100 \%$

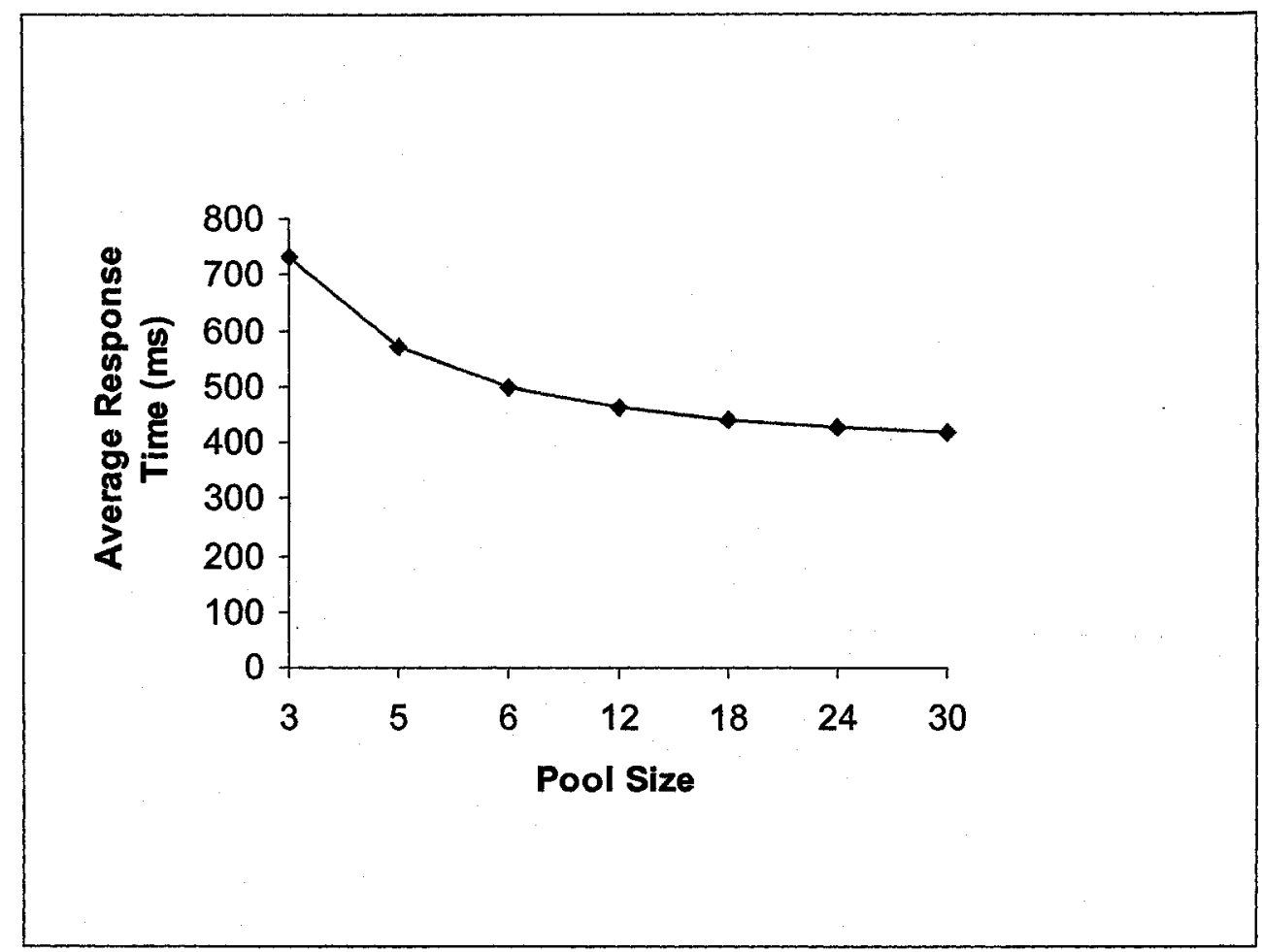

Figure 12: The effect of maximum pool size on client response time

\section{Observation:}

Figure 12 shows that the greater the pool size, the better is the mean response time. This is due to the fact that when more bean instances are available in the pool, concurrency is improved, and fewer client requests are queued. Thus the mean response time tends to improve with an increase in the maximum pool size. 


\subsubsection{The Effect of Client Request Rate $(\lambda)$ on Client Response Time, with Unequal Maximum Bean Pool Sizes for a Façade's EJBs}

This experiment will explore the situation in which a façade's EJBs maximum pool sizes are set inadequately. As such, the façade's EJBs maximum pool sizes are set inefficiently with respect to the set client request probability for each of those façade's EJBs. The intent here is to demonstrate the effect of DBPRM in situations where initially set façade's EJBs maximum pool sizes are not appropriate for the given client request probabilities.

\section{Fixed parameters for the experiment:}

- $\mathrm{S}=100$ milliseconds

Fixed for all EJBs (Bean-A, Bean-B, and Bean-C)

- DBPRM configuration for the façade's EJBs:

This is the common DBPRM configuration used for all the façade's EJBs.

$\circ \mathrm{IF}=0.5$

$\circ \mathrm{DF}=0.5$

○ $\mathrm{OT}=5$ milliseconds

Given that EJBs' service time is 100 milliseconds, this over-use threshold of 5 milliseconds would allow for an immediate DBPRM reaction for attempting to enlarge the managed maximum bean pool size of a façade's EJB, at run time.

○ $\mathrm{UT}=500$ milliseconds 
This is to prevent immediate decrease in the managed DBPRM maximum pool size for an EJB. Thus, allowing a 500 milliseconds time window, before EJB pool size is considered by the DBPRM mechanism for a decrease.

- Bean-A specific configuration:

- $\mathrm{P}=70 \%$

- $\operatorname{PMax}=3$

Given that probability of client request for this EJB was set to $70 \%$, its maximum pool size was inadequately set (low).

- Bean-B specific configuration:

- $\mathrm{P}=20 \%$

- $\quad \operatorname{PMax}=3$

Given that probability of client request for this EJB was set to $20 \%$, its maximum pool size was inadequately set (low).

- Bean-C specific configuration:

○ $\mathrm{P}=10 \%$

- $\quad \operatorname{PMax}=80$

Given that probability of client request for this EJB was set to $10 \%$, its maximum pool size was inadequately set (high).

\section{Observation:}

In this experiment the maximum pool sizes for Bean $\mathrm{A}$, Bean $\mathrm{B}$, and Bean $\mathrm{C}$ are 3, 3, and 80 . Where as the probability of requests for their bean types are $70 \%, 20 \%$, and 
$10 \%$ respectively. Such a choice of parameters is made to investigate the effect of in appropriate selection of the maximum pool size on system performance.

Figure 13 shows an improved response time when the DBPRM mechanism is activated. The improvement seems to increase with the arrival rate.

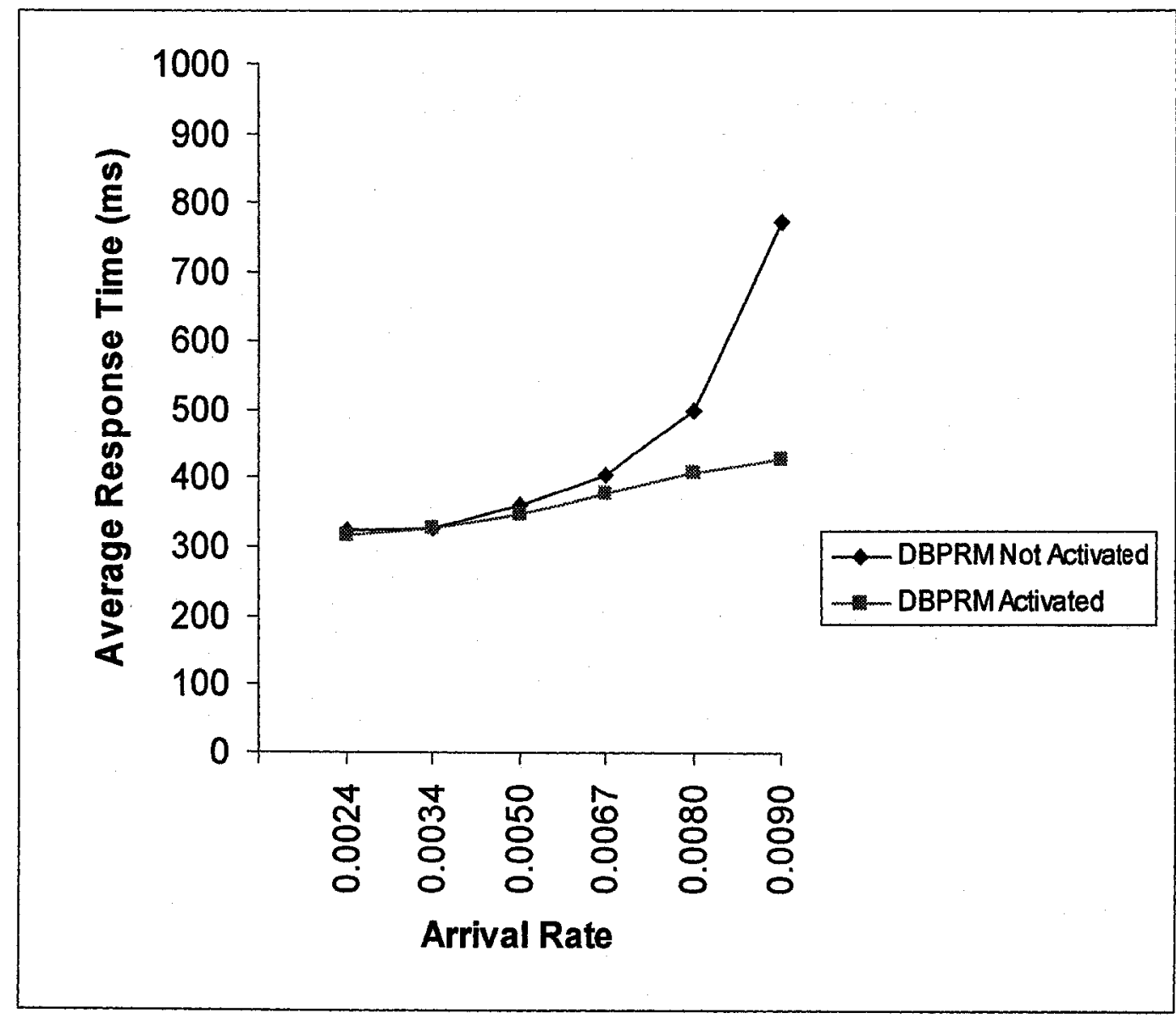

Figure 13: Effect of $\lambda$ on response time with different pool sizes in a façade's EJBs

In addition, it is important to observe that at very low $\lambda$, the DBPRM mechanism seems to yield a higher mean response time than when the mechanism is not activated. 
This is attributed to the overhead introduced by this mechanism. At smaller arrival rates, the improvement produced by the DBPRM mechanism is small and seems to be offset by this overhead.

\subsubsection{The Effect of Client Request Rate $(\lambda)$ with Equal Maximum Bean Pool}

\section{Sizes}

This experiment follows the same steps as the experiment detailed in Section 4.2.3; however Bean pool sizes are equal.

\section{Fixed parameters for the experiment:}

This experiment uses the same configurations as the experiment described in Section

4.2.3; with the exception of the following DBPRM configuration for the façade's EJBs:

- $\underline{\text { Bean-A: }}$

- $\operatorname{PMax}=\mathbf{1 0}$

- Bean-B:

○ $\quad P M a x=10$

- Bean-C:

$$
\text { ○ } \quad P M a x=10
$$

It is important to note that this experiment assumed a common maximum pool size of 10 , per façade's EJB. This value was used because we wanted the sum of all the façade's EJB maximum pool sizes to be equal to the maximum number of concurrent client requests (30) supported by the system. 


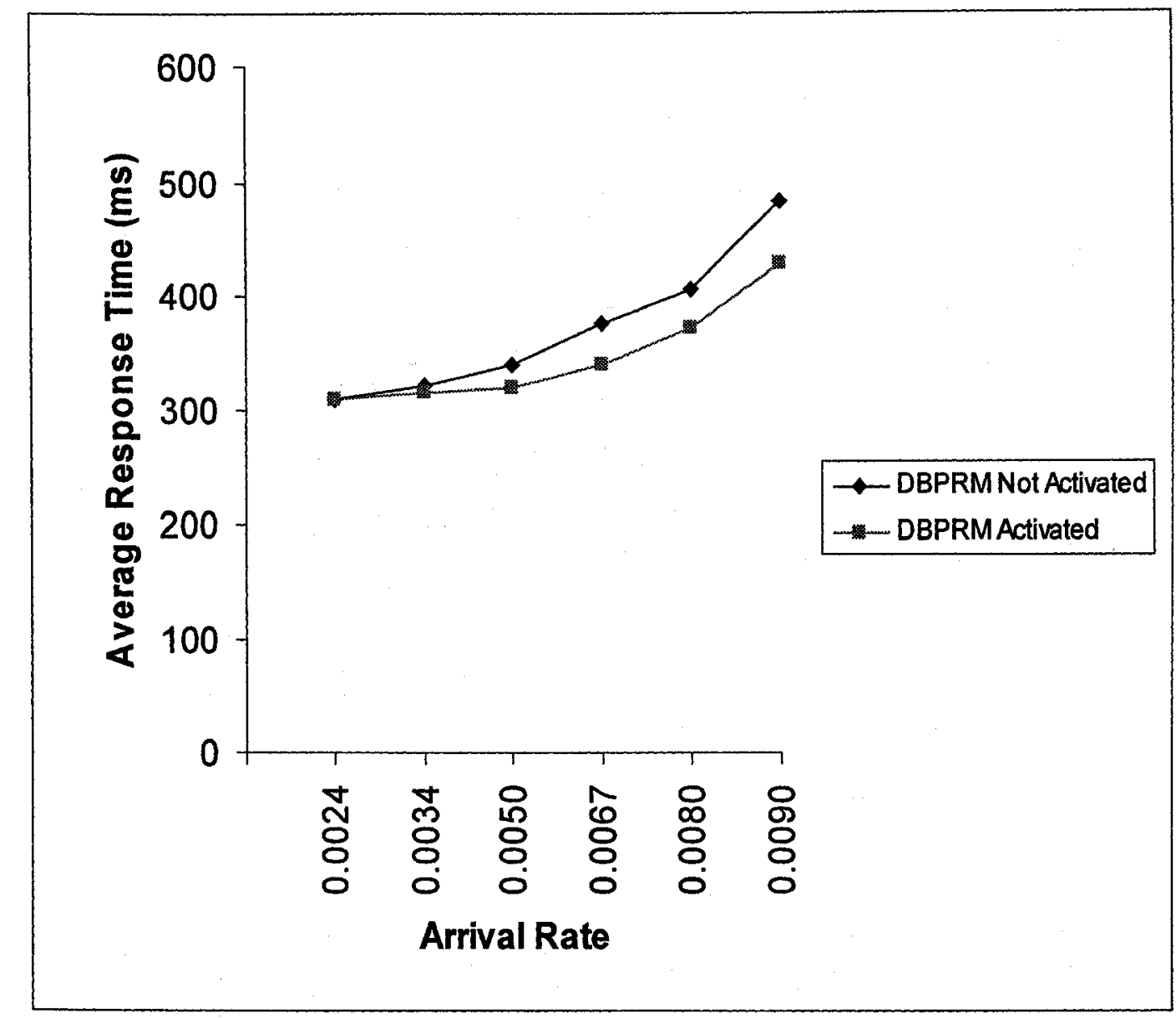

Figure 14: Effect of $\lambda$ on response time with equal maximum pool sizes for the façade's EJBs

\section{Observation:}

Similar to Figure 13, Figure 14 of this experiment shows an improved mean response time when the DBPRM mechanism is activated, at higher arrival rates. However, the improvement in this experiment is not as strong as in Figure 13. In this experiment, the maximum pool sizes were more in line with the probability of client requests for the different bean types. Thus, the base system performance without DBPRM activated is 
better than that captured in Figure 13. As a result, the improvement produced by DBPRM is smaller.

Further, Figure 15 shows the results gathered from re-running this experiment with all the façade's EJBs service times exponentially distributed. The observation remains that the DBPRM mechanism improves system performance at higher loads. Investigating system performance for higher variability in service times can form an interesting direction for future research.

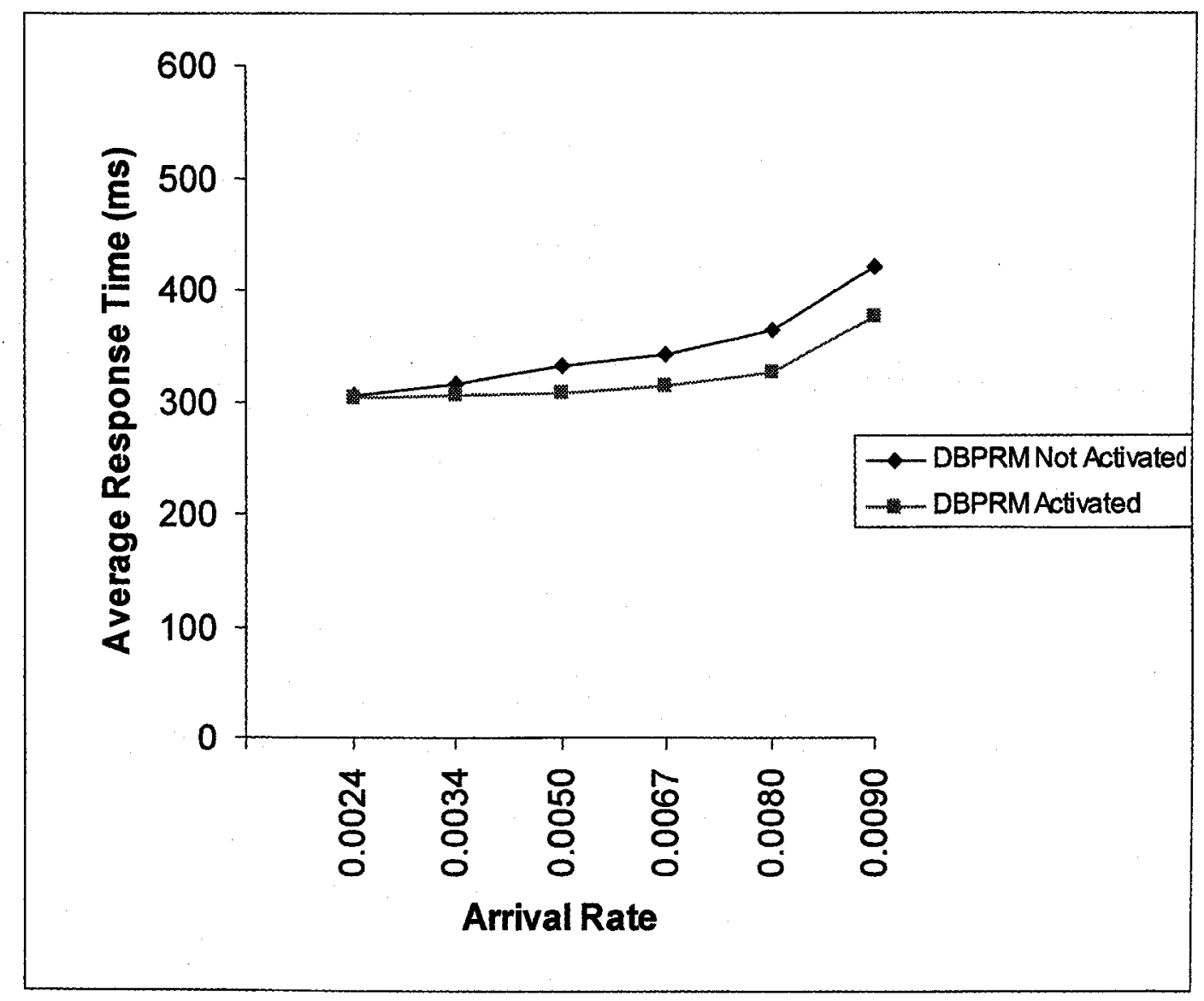

Figure 15: Effect of client request rate ( $\lambda$ ) on client's response time, with exponentially distributed service times for façade's EJBs 


\subsubsection{The Effect of Client Request Rate $(\lambda)$ with Different Service Times for}

\section{EJBs}

This experiment will explore the effect of different service times for the façade's EJBs on mean response time. All the façade's EJBs service times have been set based on the following:

- The façade's EJB with the highest configured request probability (i.e. $70 \%$ ) has the lowest service time, while the façade's EJB with the lowest configured client request probability (i.e. 10\%) has the highest service time.

\section{Fixed parameters for the experiment:}

This experiment uses the same parameters as the experiment described in Section 4.2.4, with the exception of the following parameters for the façade's EJBs:

- Bean-A:

$S($ Fixed $)=75$ milliseconds

- Bean-B:

$\mathrm{S}($ Fixed $)=100$ milliseconds

- Bean-C:

$\mathrm{S}($ Fixed $)=125$ milliseconds

\section{Observation:}

The observations made from Figure 16 are similar to the ones noted for Figure 13. As such, it continues to be observed that mean response time improves at higher arrival rates when the DBPRM mechanism is activated. 


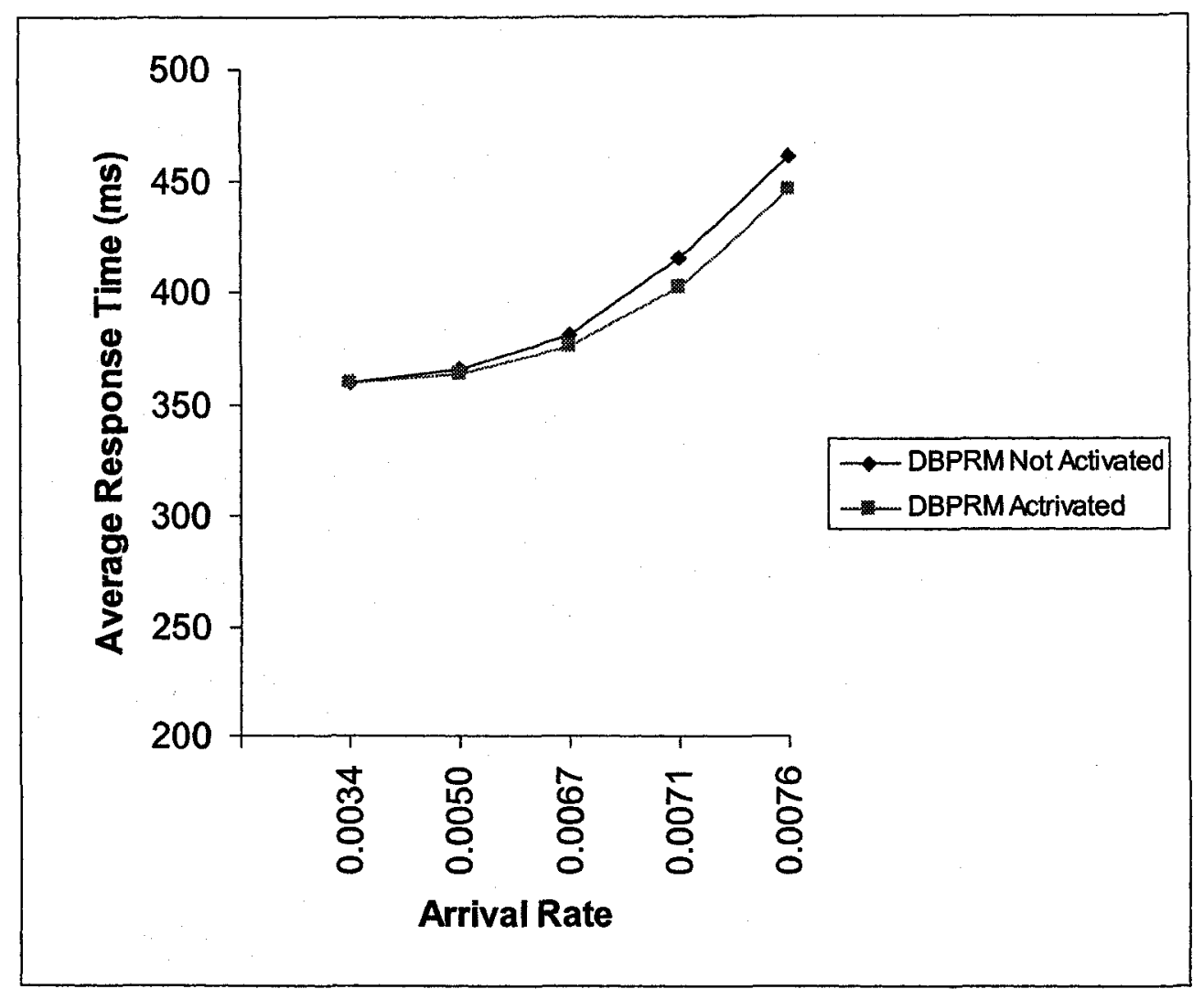

Figure 16: The effect of $\lambda$ on response time with different fixed service time configurations for a façade's EJBs

\subsubsection{The Effect of the DBPRM Over-use Time Threshold}

This experiment analyzes the effect of the DBPRM's over-use time threshold on system performance. The over-use time threshold is the variable factor, and all façade's EJBs share the same over-use time threshold.

\section{Fixed parameters for the experiment:}

This experiment uses the same configurations as the experiment described in Section 4.2.4, with the exception of the following: 
- $\quad$ IAT $=110$ milliseconds

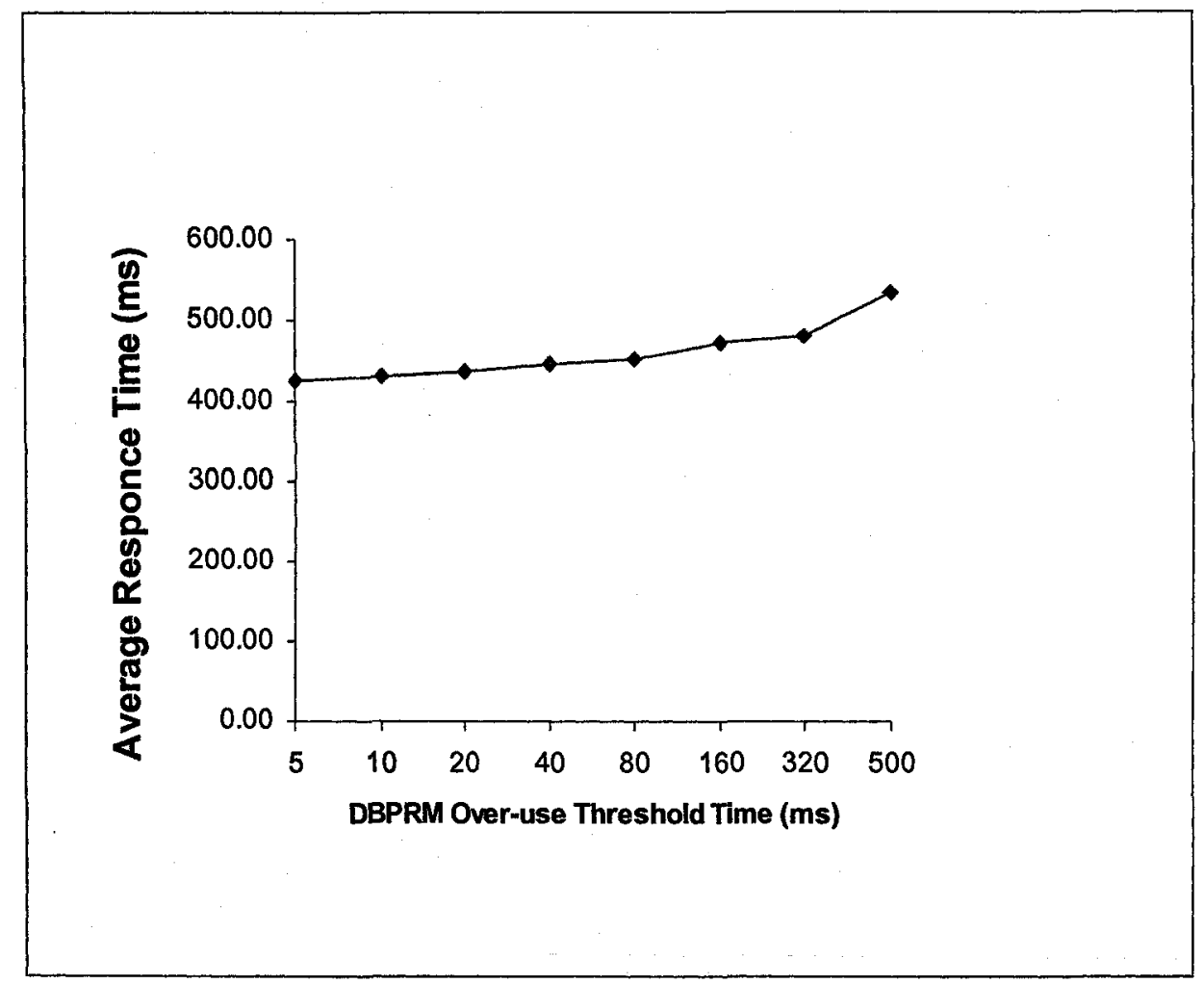

Figure 17: Effect of DBPRM over-use time threshold on client response time

\section{Observation:}

This experiment shows the effect of the DBPRM over-use threshold on mean response time. The higher this threshold, the longer the client request will have to be queued before the DBPRM mechanism allows for resources to become available (reconfigure appropriate maximum pool sizes). Figure 17 shows deterioration in system performance with an increase in DBPRM over-use threshold time. This is because the higher the over-use time threshold, the longer is the delay for the DBPRM mechanism 
to take effect in adapting the required maximum pool size of the façade's EJBs to the client request demand.

\subsubsection{The Effect of Service Time for Façade's EJBs, while DBPRM Activated}

This experiment aims to compare the effect on system performance of different fixed service time settings for a façade's EJBs, while DBPRM is activated. The objective here is to compare system performance based on different variations of fixed service times, while maintaining the same client request probability for each bean type in the façade.

This experiment depends on three sub-experiment runs; each with a specific set of values for service times. A bar chart shows a comparison of the results of each of those subexperiments.

\section{Fixed parameters for the experiment:}

This experiment uses the same configuration as in the experiment described in Section 4.2.4, with the exception of the following:

- $\quad$ IAT $=110$ milliseconds

- In all sub-experiments, the façade's EJBs fixed service time configurations and request probabilities were selected in such a way that an overall mean service time (S) of 100 milliseconds is achieved.

- Table 1 shows additional set parameters specific to each performed subexperiment: 
Table 1: Additional fixed parameters for the effect of fixed service time on response time, with DBPRM activated experiment

\begin{tabular}{|l|l|l|l|}
\cline { 2 - 4 } \multicolumn{1}{c|}{} & Sub-experiment 1 & Sub-experiment 2 & Sub-experiment 3 \\
\hline Bean-A & $\mathrm{P}=70 \%$ & $\mathrm{P}=70 \%$ & $\mathrm{P}=70 \%$ \\
& $\mathrm{~S}=86$ milliseconds & $\mathrm{S}=100$ milliseconds & $\mathrm{S}=120$ milliseconds \\
\hline Bean-B & $\mathrm{P}=20 \%$ & $\mathrm{P}=20 \%$ & $\mathrm{P}=20 \%$ \\
& $\mathrm{~S}=100$ milliseconds & $\mathrm{S}=100$ milliseconds & $\mathrm{S}=50$ milliseconds \\
\hline Bean-C & $\mathrm{P}=10 \%$ & $\mathrm{P}=10 \%$ & $\mathrm{P}=10 \%$ \\
& $\mathrm{~S}=200$ milliseconds & $\mathrm{S}=100$ milliseconds & $\mathrm{S}=60$ milliseconds \\
\hline
\end{tabular}

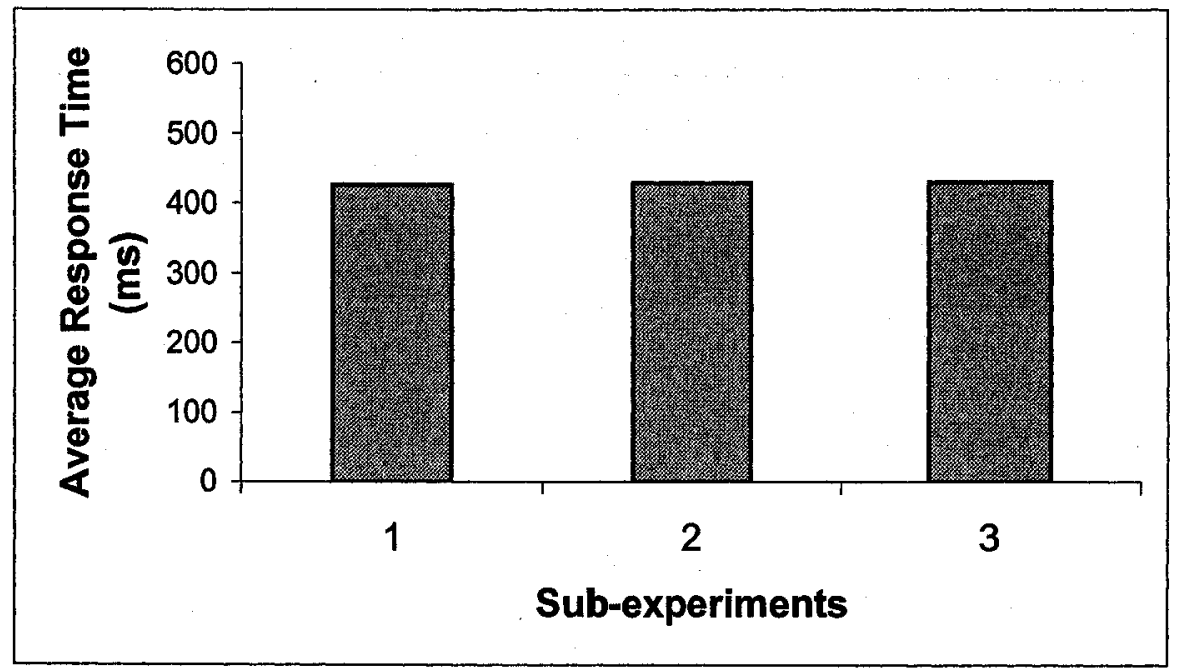

Figure 18: Effect of fixed service time on client response time, with DBPRM activated

\section{Observation:}


Figure 18 demonstrates that comparable mean client response times are achieved in all the sub-experiments. Thus, the performance of DBPRM seems to be insensitive to the values used for the mean service times of the individual bean types, as long as the overall mean service time remains the same.

\subsubsection{The Effect of Client Request Probability on Client Response Time}

This experiment investigates the effect of client request probability on system performance, with both DBPRM activated and not activated. This effect is examined under different sets of client request probability configurations for the façade's EJB types. Each set of client request probability configuration is examined in a separate subexperiment.

\section{Fixed parameters for the experiment:}

This experiment uses the same configurations as in the experiment described in Section 4.2.4, with the exception of the following:

- $\mathrm{IAT}=110$ milliseconds (For all bean types)

- $\mathrm{S}=100$ milliseconds (For all bean types)

- Each of the following sub-experiments is composed of two experiment runs, one with DBPRM activated, and the other without DBPRM activated.

- Table 2 shows additional set parameters specific to each performed subexperiment: 
Table 2: Additional fixed parameters for the effect of client request probability on client response time experiment

\begin{tabular}{|l|l|l|l|}
\cline { 2 - 4 } \multicolumn{1}{c|}{} & Sub-experiment 1 & Sub-experiment 2 & Sub-experiment 3 \\
\hline Bean-A & $\mathrm{P}=70 \%$ & $\mathrm{P}=50 \%$ & $\mathrm{P}=33.33 \%$ \\
\hline Bean-B & $\mathrm{P}=20 \%$ & $\mathrm{P}=30 \%$ & $\mathrm{P}=33.33 \%$ \\
\hline Bean-C & $\mathrm{P}=10 \%$ & $\mathrm{P}=20 \%$ & $\mathrm{P}=33.33 \%$ \\
\hline
\end{tabular}

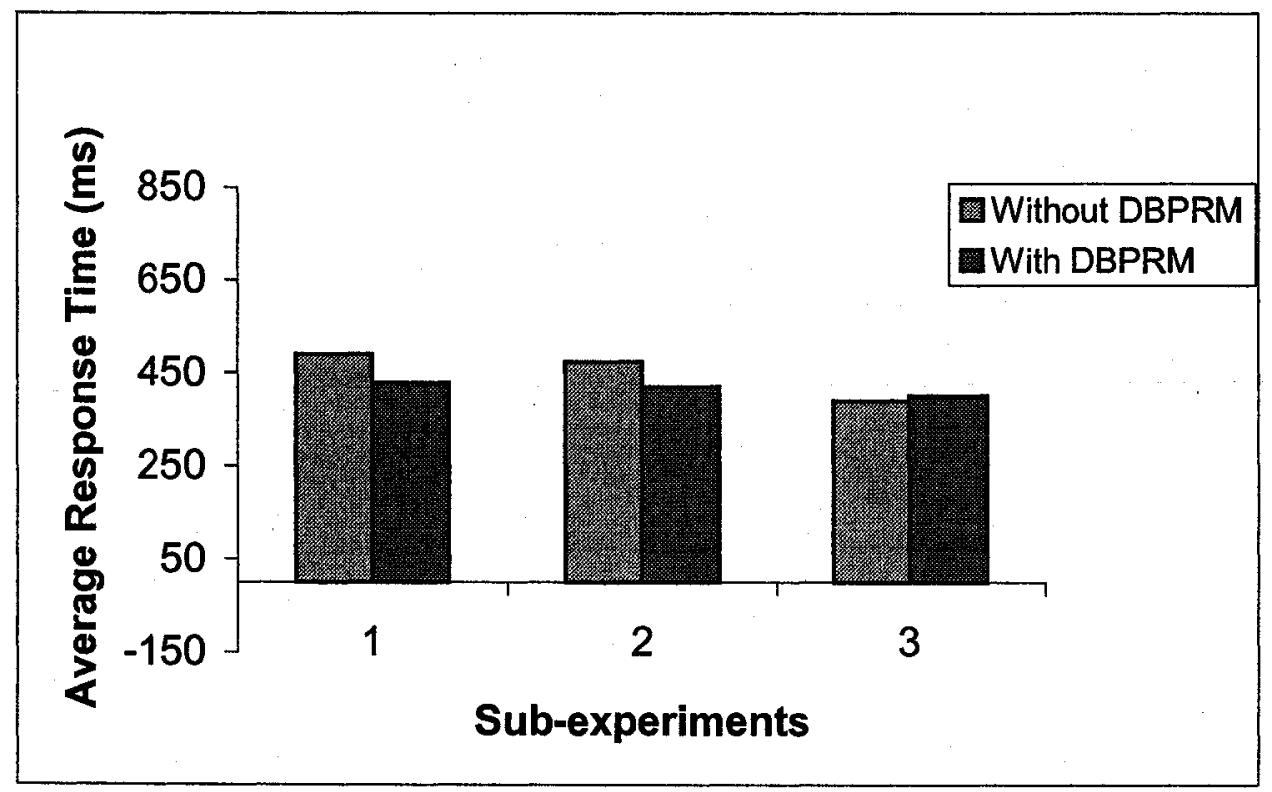

Figure 19: Effect of client request probability on client response time

\section{Observation:}

Figure 19 shows that the results for Sub-experiment 1 and Sub-experiment 2 are similar. The DBPRM mechanism leads to a significant improvement in performance. 
However, in Sub-experiment 3, the overall mean response time is slightly higher when DBPRM is activated. This is attributed to the fact that Sub-experiment 3 had equal client request probabilities of $33.33 \%$ for all of its façade's bean types. As such, considering that the maximum number of concurrent clients in this experiment was 30 , the number of concurrent client requests for a given bean type would tend to be accommodated with the initially set pool size (PMax $=10)$. Thus, the impact of queued client request was not visible, when compared with Sub-experiment 1 or Subexperiment2, for the case where DBPRM was not activated. Thus, the benefit due to DBPRM was small and was offset by the DBPRM mechanism's overhead in Subexperiment 3.

\subsubsection{The Effect of DBPRM Under-use Time Threshold}

This experiment analyzes the effect of DBPRM's under-use time threshold on system performance. The under-use time threshold is used as a variable factor, and all the façade's EJBs share the same over-use time threshold.

\section{Fixed parameters for the experiment:}

This experiment uses the same configurations as the experiment described in Section 4.2.4, with the exception of the following:

- IAT $=110$ milliseconds (For all façade's bean types) 


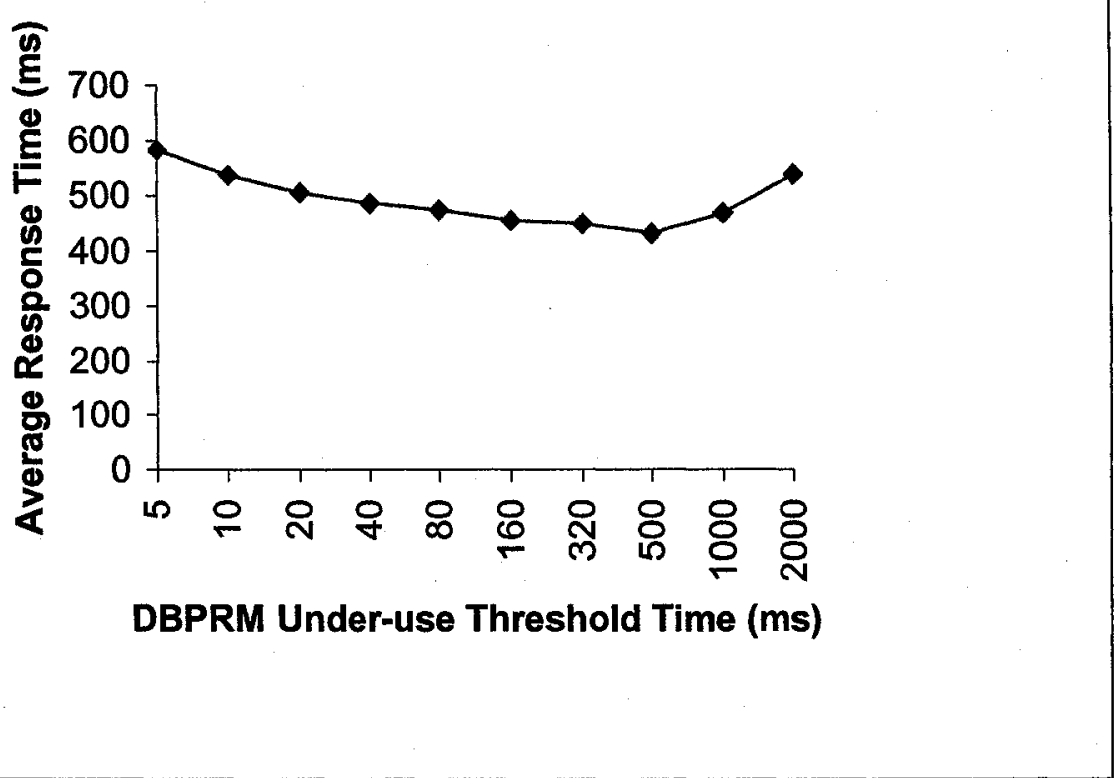

Figure 20: Effect of DBPRM under-use time threshold on client response time

\section{Observation:}

Figure 20 shows the effect of the DBPRM under-use threshold on mean response time. Under-use threshold configuration controls the time that the DBPRM mechanism has to wait before decreasing a pool size for one bean type to in the EJB façade. See Section 3.3 for details on the under-use threshold usage of the DBPRM mechanism.

The average response time tends to decrease with an increase in the under-use time threshold. However, if this threshold is increased beyond 500 milliseconds, average response time seems to increase. The rationale for this behavior is provided. System behavior at high under-use threshold values is explained first, followed by a discussion of the system behavior at low under-use threshold values. 
At very high values of this threshold, the DBPRM mechanism takes a long time to be able to adapt the pool sizes of the under used bean types (i.e. bean types with a low number of client requests). Thus, concurrent client requests of an over used bean type will be queued longer for the DBPRM mechanism to be activated and enhance its pool size.

Further, Figure 20 also shows that setting this threshold too low leads to degradation in the overall mean response time of the experiment. This is due to the premature adaptation of under used bean's pool size (i.e. bean type with a lower number of client requests). Due to this premature adaptation based on a temporary low number of client requests, the requests that arrive later will be queued for a longer period of time to receive service. Thus, intermediate range of values seems to be appropriate for the under-use time threshold.

\subsubsection{The Effect of Static Maximum Pool Size, versus the Effect of DBPRM's Dynamic Adaptation to Maximum Pool Size.}

This experiment analyzes the performance of a statically configured system in which the maximum pool sizes of the façade's EJBs were fixed and in line with the proportion of client requests for each façade's EJB. The performance of the statically configured system is then compared with results from the experiment described in Section 4.2.7, where the DBPRM mechanism was activated.

\section{Fixed parameters for the experiment:}


The effect of fixed service times for a façade's EJBs has been analyzed based on the following three sub-experiments. All the sub-experiments carried out use the same configurations as the sub-experiments detailed in Section 4.2.7, with the exception of the following:

- DBPRM is not activated.

- Table 3 shows additional set parameters specific to each performed subexperiment:

Table 3: Additional fixed parameters for the Comparison between a statically configured system and a DBPRM activated system experiment

\begin{tabular}{|c|c|c|c|}
\hline & Sub-experiment $1^{6}$ & Sub-experiment 2 & Sub-experiment 3 \\
\hline Bean-A & $\begin{array}{l}\mathrm{P}=70 \% \\
\mathrm{~S} \text { (Fixed) }=86 \mathrm{~ms} \\
\mathrm{PMax}=21\end{array}$ & $\begin{array}{l}\mathrm{P}=70 \% \\
\mathrm{~S} \text { (Fixed) }=100 \mathrm{~ms} \\
\mathrm{PMax}=21\end{array}$ & $\begin{array}{l}\mathrm{P}=70 \% \\
\mathrm{~S} \text { (Fixed) }=120 \mathrm{~ms} \\
\mathrm{PMax}=21\end{array}$ \\
\hline Bean-B & $\begin{array}{l}\mathrm{P}=20 \% \\
\mathrm{~S} \text { (Fixed) }=100 \mathrm{~ms} \\
\mathrm{PMax}=6\end{array}$ & $\begin{array}{l}\mathrm{P}=20 \% \\
\mathrm{~S}(\text { Fixed) }=100 \mathrm{~ms} \\
\mathrm{PMax}=6\end{array}$ & $\begin{array}{l}\mathrm{P}=20 \% \\
\mathrm{~S}(\text { Fixed })=50 \mathrm{~ms} \\
\mathrm{PMax}=6\end{array}$ \\
\hline Bean-C & $\begin{array}{l}\mathrm{P}=10 \% \\
\mathrm{~S} \text { (Fixed) }=200 \mathrm{~ms} \\
\mathrm{PMax}=3\end{array}$ & $\begin{array}{l}\mathrm{P}=10 \% \\
\mathrm{~S} \text { (Fixed) }=100 \mathrm{~ms} \\
\mathrm{PMax}=3\end{array}$ & $\begin{array}{l}\mathrm{P}=10 \% \\
\mathrm{~S} \text { (Fixed) }=60 \mathrm{~ms} \\
\mathrm{PMax}=3\end{array}$ \\
\hline
\end{tabular}

\footnotetext{
${ }^{6}$ The maximum pool size for a particular bean type was obtained as a product of the probability of request for that bean type and the maximum number of concurrent client requests on the system that was fixed at 30 (See Section 4.2.1)
} 


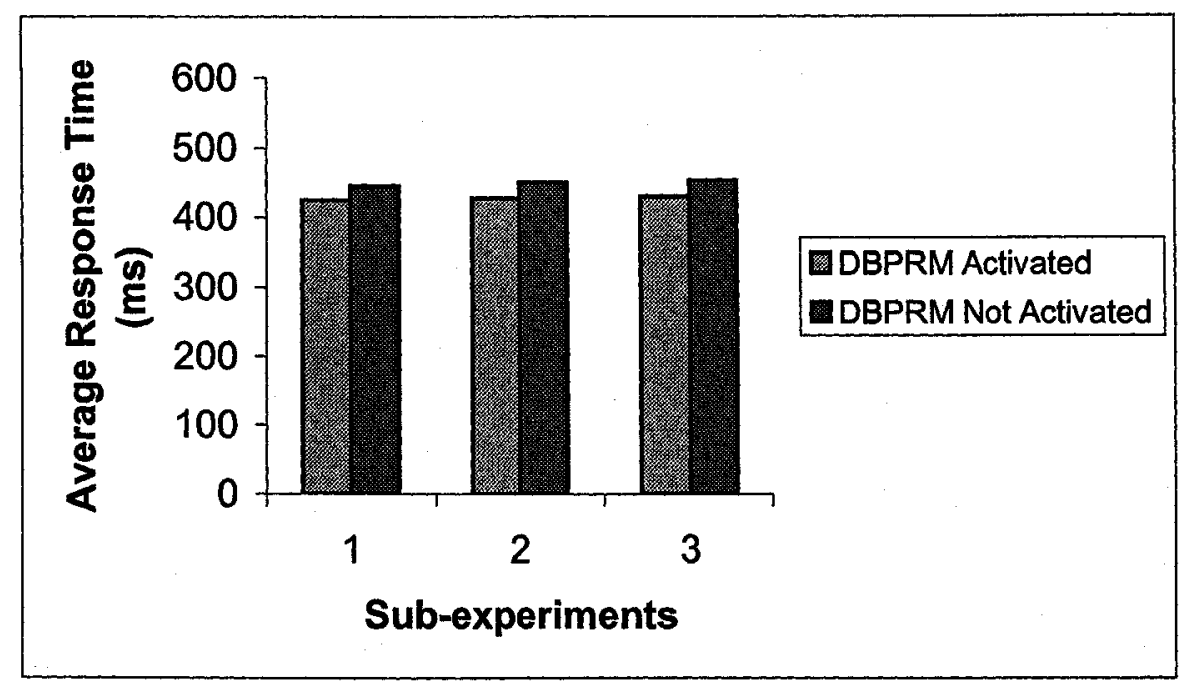

Figure 21: Comparison between a statically configured system and a DBPRM activated system.

Note that the results for the DBPRM activated system are repeated from Section 4.2.7

\section{Observation:}

Figure 21 shows that the sub-experiments carried out and described in this section yielded comparable mean response time for a given system. We note that a fixed static configuration of the façade's EJBs maximum pool size yields a higher system response time, in comparison to when the DBPRM mechanism is activated. This helps demonstrate the advantage of the DBPRM mechanism that adapts to the system state. Thus, system performance achieved using a fixed static pool size configuration, which was based on the known probability of client requests to the different façade's EJBs, is inferior to the performance observed in the system when DBPRM is activated. 
Further, the following figures (Figure 22and Figure 23) show the results gathered using the same configuration as in sub-experiment 1 , but with different $\lambda \mathrm{s}$. Figure 22 shows the results observed when the façade's EJBs service times were fixed, as opposed to being exponentially distributed, as in Figure 23.

Both of the following figures provide further illustration that the DBPRM mechanism can still improve system performance as the arrival rate of client requests increases.

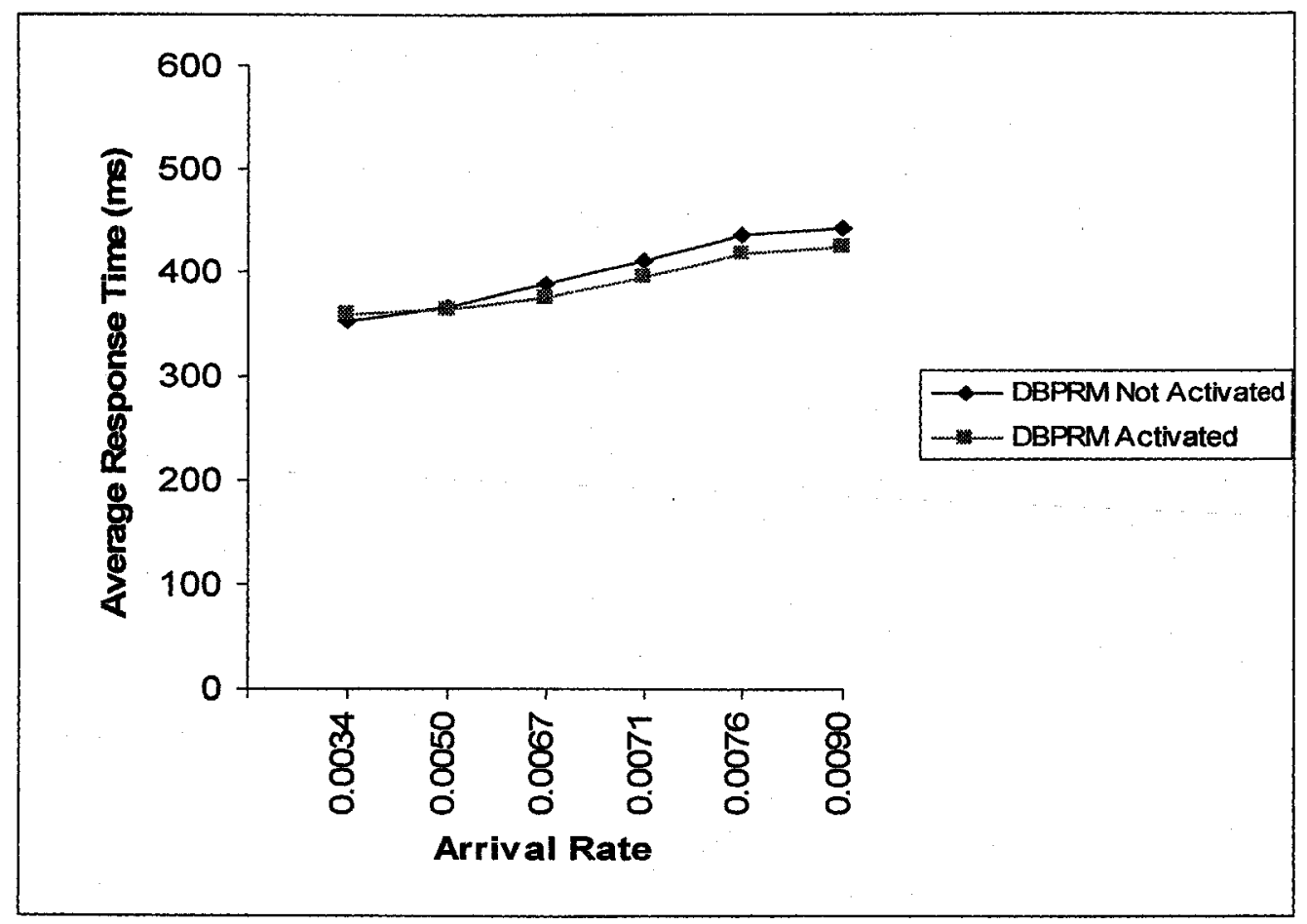

Figure 22: The effect of DBPRM's dynamic adaptation of maximum pool size (service time is fixed) 


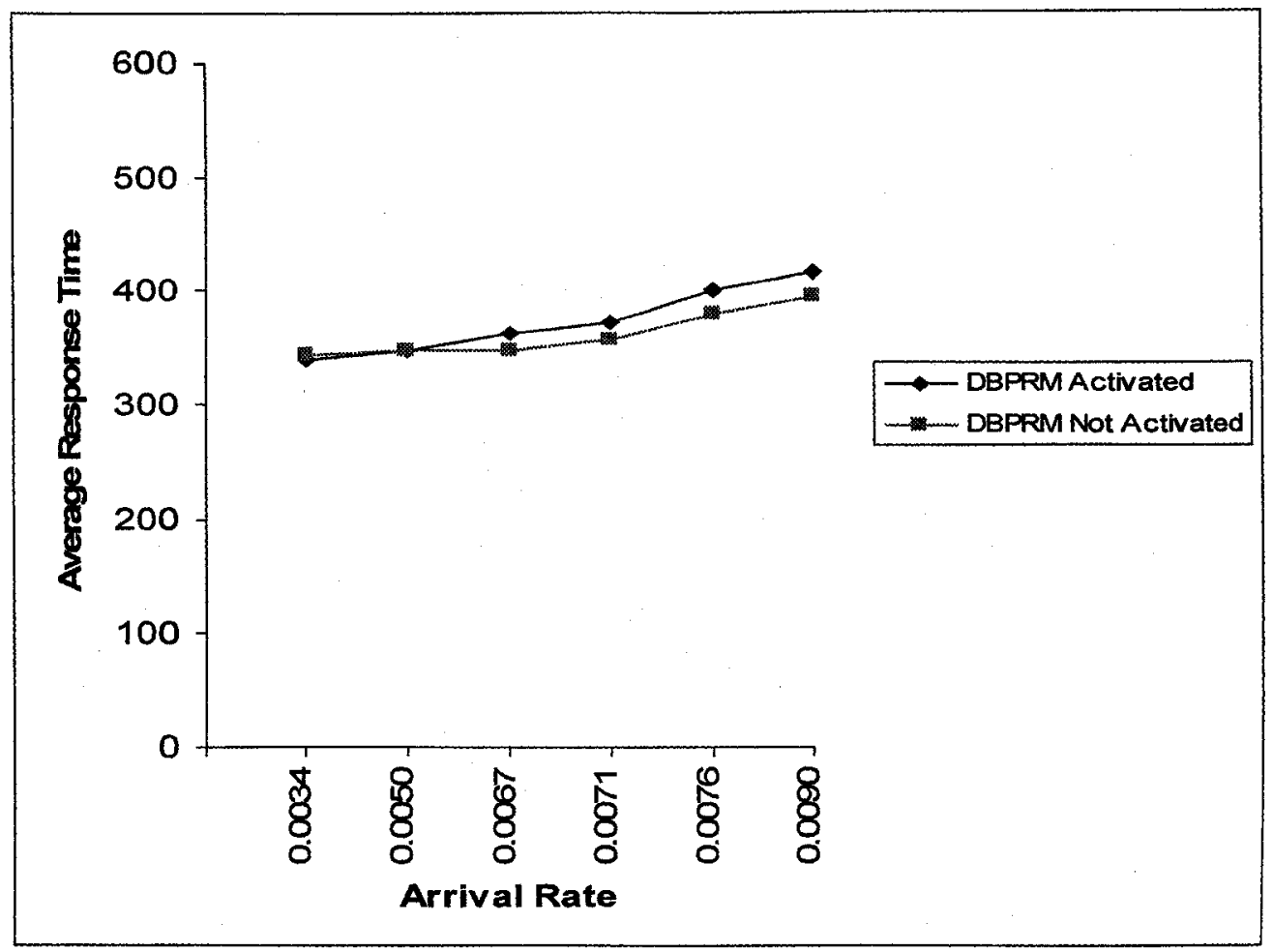

Figure 23: The effect of DBPRM's dynamic adaptation of maximum pool size (service time is exponentially distributed)

\subsubsection{The Effect of DBPRM Increase/Decrease Factors}

This experiment aims to analyze the effect of DBPRM increase/decrease factors on system performance. The experiment will test system performance based on low, medium, and high DBPRM increase/decrease factors for all systems' façade's EJBs.

This experiment depends on three sub-experiment runs. Each sub-experiment is run with a particular DBPRM increase/decrease factor configuration.

\section{Fixed parameters for experiments:}

This experiment uses the same configurations as the experiment detailed in Section 4.2.4, with the exception of the following: 
- $\mathrm{IAT}=110$ milliseconds

- DBPRM was activated for all sub-experiments.Table 4 shows additional set parameters specific to each performed sub-experiment:

Table 4: Additional fixed parameters for the effect of DBPRM Increase/Decrease Factors on Client Mean Response Time experiment

\begin{tabular}{|l|l|l|l|}
\cline { 2 - 4 } \multicolumn{1}{c|}{} & Sub-experiment 1 & Sub-experiment 2 & Sub-experiment 3 \\
\hline & $\mathrm{P}=70 \%$ & $\mathrm{P}=70 \%$ & $\mathrm{P}=70 \%$ \\
$\mathrm{IFean}-\mathrm{A}$ & $\mathrm{IF}=0.25$ & $\mathrm{IF}=0.50$ & $\mathrm{IF}=0.75$ \\
& $\mathrm{DF}=0.25$ & $\mathrm{DF}=0.50$ & $\mathrm{DF}=0.75$ \\
\hline Bean-B & $\mathrm{P}=20 \%$ & $\mathrm{P}=0.20 \%$ & $\mathrm{P}=20 \%$ \\
& $\mathrm{DF}=0.25$ & $\mathrm{IF}=0.50$ & $\mathrm{DF}=0.75$ \\
\hline \multirow{2}{*}{ Bean-C } & $\mathrm{P}=10 \%$ & $\mathrm{DF}=0.50$ & $\mathrm{P}=10 \%$ \\
& $\mathrm{IF}=0.25$ & $\mathrm{IF}=0.50$ & $\mathrm{IF}=0.75$ \\
& $\mathrm{DF}=0.25$ & $\mathrm{DF}=0.50$ & $\mathrm{DF}=0.75$ \\
\hline
\end{tabular}

\section{Observation:}

The impact of the DBPRM increase/decrease factor on system performance seems to be very modest (see Figure 24). The best performance achieved was for a medium level of DBPRM increase/decrease factor. This is due to the fact that with a low 


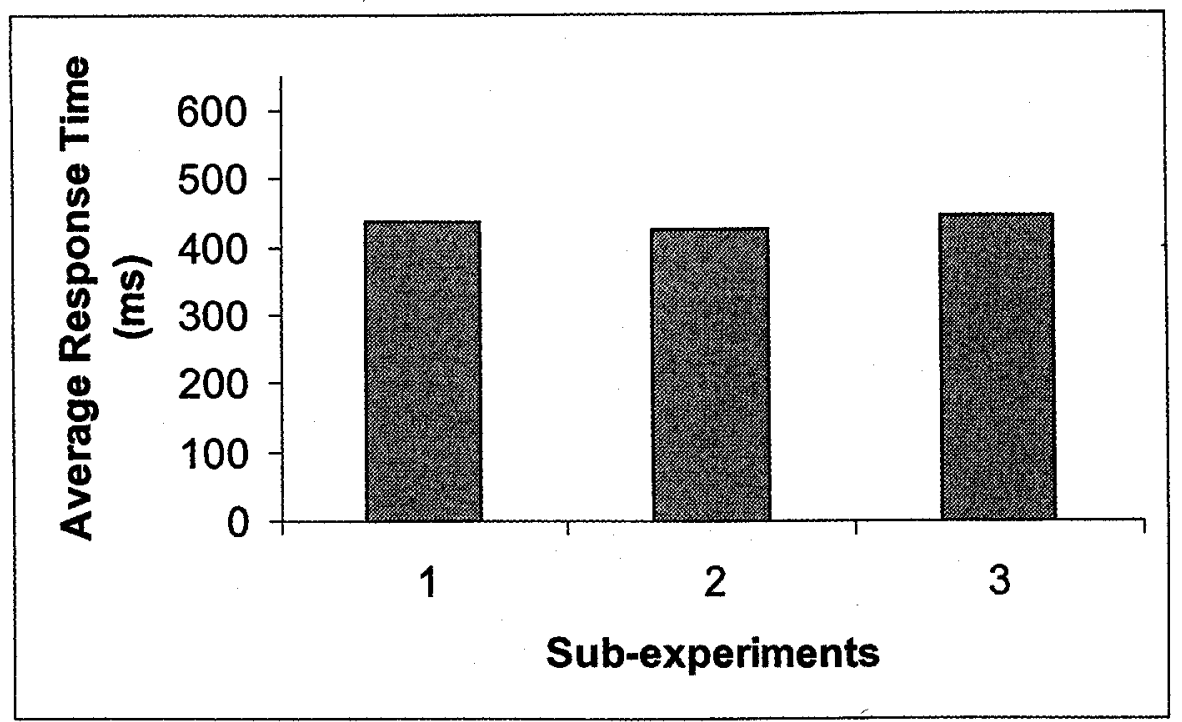

Figure 24: Effect of DBPRM Increase/Decrease Factors on Client Mean Response Time

DBPRM increase/decrease factor, the DBPRM mechanism will take longer to appropriately adapt the pool sizes for the façade's EJBs that have higher number of client requests. On the other hand, at a high DBPRM increase/decrease factor, the DBPRM mechanism will increase the pool sizes for the façade's EJBs that have higher number of client requests, at a high rate and at the expense of other façade's EJBs with lower number of client requests. This may cause a premature reallocation of pool size for the façade's EJBs with lower number of client requests. As a result, the requests arriving in the near future may need to wait longer for receiving service. However the difference in the average response times observed for the different sub-experiments was very small. 


\subsubsection{The Effect of a Low Client Request Rate}

This experiment analyzes the effect of a very low $\lambda$ on system performance. The experiment depends on two sub-experiments runs. One sub-experiment was run with DBPRM not activated, while the other was run with DBPRM activated. The objective here was to compare system performance achieved in both sub-experiments in order to understand the effect of DBPRM's overhead on system performance, when $\lambda$ is low and DBPRM is the least effective.

\section{Specific configurations for the experiments:}

This experiment uses the same configurations as the experiment described in Section 4.2.4, with the exception of the following:

- $\quad \mathrm{IAT}=1000$ milliseconds

- In all the sub-experiments, the façade's EJBs fixed service time (S) configurations were selected in such a way that an overall mean service time of 100 milliseconds was maintained for each sub-experiment.

- Sub-experiment 1 was run with DBPRM not activated, while Sub-experiment 2 was run with DBPRM activated. The following are the façade's EJB configurations used for both sub-experiments:

$\underline{\text { Bean }-\mathrm{A}}$

- $\mathrm{P}=70 \%$

- $\mathrm{S}$ (Fixed) $=86$ milliseconds.

Bean - B

- $\mathrm{P}=20 \%$ 
- $\quad \mathrm{S}($ Fixed $)=100$ milliseconds.

$\underline{B e a n-C}$

- $\mathrm{P}=10 \%$

- $\quad \mathrm{S}($ Fixed $)=200$ milliseconds.

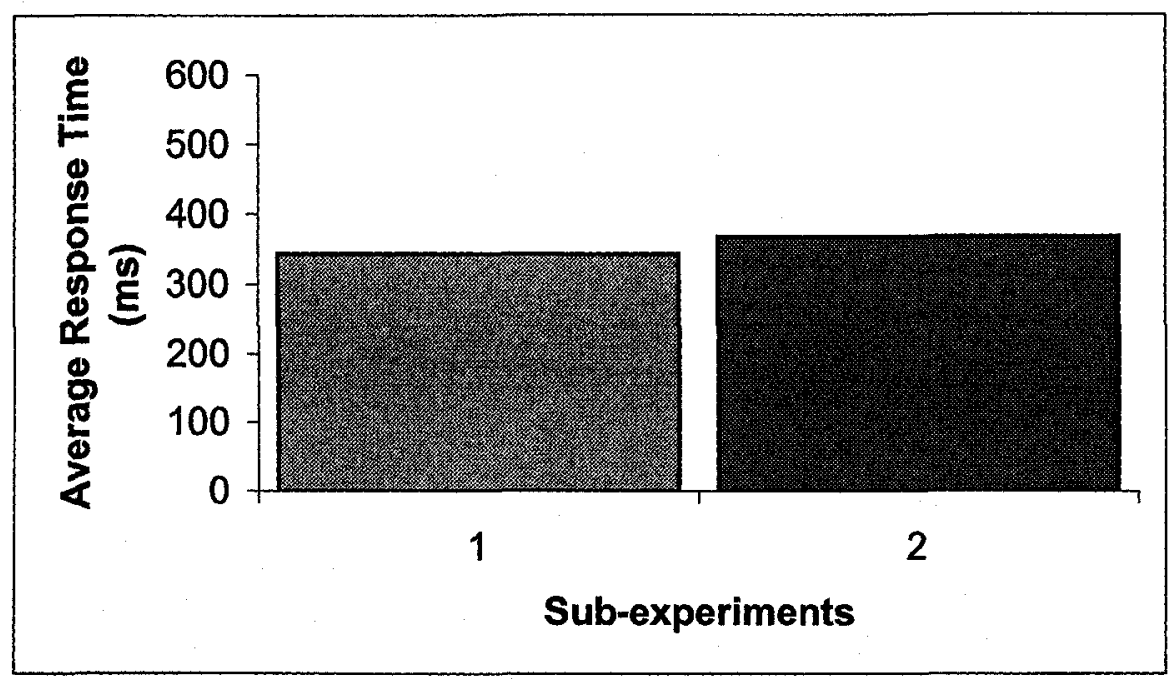

Figure 25: Effect of low client request rate $(\lambda)$ on client response time

\section{Observation:}

Figure 25 shows that at a very low $\lambda$, the system with the DBPRM mechanism activated, yields a slightly higher mean response time than when the mechanism is not activated. This is attributed to the overhead introduced by the DBPRM mechanism. When client request rate is very low, there is not much contention for system resources, and the DBPRM mechanism is least effective. So the effect of overhead dominates, and system performance with DBPRM activated is slightly inferior to the system on which DBPRM was not activated. 


\section{Chapter 5 Conclusions}

\subsection{Summary}

The main focus of the thesis was the design and implementation of an adaptive Web application-based J2EE EJB pool size configuration control mechanism, and an analysis of its performance. This mechanism enhances Web-based application performance by dynamically adapting the stateless-session façade's EJBs' maximum pool sizes to their corresponding client request load, at run time. Descriptions of the experiments performed, as well as an interpretation of the results, were presented in Chapter 4.

\subsection{Insights Gained}

Finding an effective pool size depends on a number of factors, including the service time of client requests and the potential limitation in resources to which clients could be sharing access via their EJB tier, such as a database [36]. In addition, effective pool size configuration depends directly on the number of clients requests, which is difficult to predict, especially in Web-based environments, where the number of clients requests tends to be very dynamic. When the DBPRM mechanism is used, it was demonstrated that its dynamic adaptive control of the façade's EJBs pool sizes allows for an enhanced overall client response time, in comparison to that obtained with a static configuration of fixed pool sizes for façade's EJBs, done at the time when the façade's EJBs are deployed. 
Careful set-up and understanding of these configurations is required for this mechanism to be used effectively. The following are the key observations made:

- Section 4.2.6 showed that the higher the DBPRM mechanism's over-use time threshold for a controlled EJB pool size, the longer the delay for the DBPRM mechanism to take effect in adapting its maximum pool size to its client request load. Thus, having the over-use time threshold set to a low value seems to improve system performance.

- Section 4.2.9 showed that the higher the DBPRM mechanisms' under-use time threshold, the longer requests will have to be queued before the DBPRM mechanism allows for the required pool size adaptation. This can cause increased queuing time for those façade's EJBs with higher client requests load than their existing pool size configuration can handle.

Further, setting this threshold too low will lead to degradation in client response times, due to the premature adaptation of EJB pool sizes with lower number of client requests, and depriving the EJBs that are subject to higher client request loads. Thus, using an intermediate value for this parameter seems appropriate (See Figure 20 for example).

- Section 4.2.11 showed that the best performance achieved is for a medium level of DBPRM increase/decrease factor in the system. 
Note that the appropriate values of the DBPRM control parameters may be dependent on the system and the workload. Thus, some initial experimentation may be necessary to determine these values.

Further, Sections 4.2.8 and 4.2.12 showed the deterioration in performance caused by the DBPRM mechanism's overhead when the client request load is already being managed reasonably by the existing static configuration of bean pool sizes. This overhead is the cost associated with DBPRM. However, observed improvement in system performance out-weighs the draw-back of DBPRM's overhead, when there is more variation in the number of client requests for the different bean types.

\subsection{Thesis Contributions}

To the best of our knowledge, there has been little research on adaptive self-configuration for J2EE application servers. An investigation of good application server configurations using an empirical approach is presented in [16]. However, the authors only provide a rule of thumb for manually configuring application servers. The framework proposed by [2] focuses on J2EE applications, not on J2EE application servers, and their application adaptation is still performed by an admin managing the application. With J2EE-based middleware architecture, the need to address its application server (resources) configuration in an adaptive manner is important [26].

This thesis introduces a technique for dynamically controlling the bean pool size of a Web-based application. The pool size is adaptively altered in response to a change in the 
number of clients' requests. The design and prototype implementation of the DBPRM mechanism were presented, and its performance under various workload conditions was thoroughly analyzed. A number of insights into system performance were obtained from this performance analysis. The thesis also included the design and implementation of a URL performance analyzer used to analyze the performance of the DBPRM mechanism.

\subsection{Future Work}

This research opens the door for a number of future research opportunities:

- An enhanced implementation of the DBPRM mechanism by introducing a software agent-based intelligent algorithm to monitor and control the technique. Such intelligence would allow for the DBPRM mechanism to learn and predict client request patterns, and thus be able to efficiently reconfigure the pool sizes. The intelligence of this algorithm can be implemented using an if-then reasoning approach, or through the implementation of an Expert System reasoning algorithm. Expert System is a rule-based processing system. It consists of three major elements: a knowledge base (the set of if-then rules and known facts), a working memory or database of derived facts and data, and an inference engine, which contains the reasoning logic used to process the rules and data [6]. The following are two candidate expert system algorithms that have available implementations, and can be adopted to fit the use of our EJB monitoring/optimization algorithm:

- Expert Systems Reasoning Using Forward Chaining Algorithm: 
Forward chaining is a data-driven reasoning process in which a set of rules is used to derive new facts from an initial set of data. This algorithm is used in real-time monitoring and diagnostic systems where quick identification and response to problems are required [6].

- Expert Systems Reasoning Using the Jess system:

Jess is a tool for building a type of intelligent Expert System software. It uses a special algorithm called Rete to match the rules to the facts. Rete makes Jess much faster than a simple set of cascading if- then statements in a loop. Jess was originally conceived as a Java clone of CLIPS (C Language Integrated Production System), but nowadays has many features that differentiate it from its parent [6]. 


\section{References}

[1] T. Abdelzaher, N. Bhatti, "Web Server QoS Management by Adaptive Content Delivery", in Proceedings of the $7^{\text {th }}$ International Workshop on Quality of Service, London, UK, May 1999, pp. 216-225.

[2] M. A. Aleksandrov, V. S. Voinov, "Designing and implementing QoS management of the Web". in Proceedings of 1998 conference of the Centre for Advanced Studies on Collaborative Research, Toronto, Canada, November 1998, pp. 21-34.

[3] D. Alur, J. Crupi, D. Malks, "Core J2EE PATTERNS: Best Practices and Design Strategies (2 $2^{\text {nd }}$ Edition)", Prentice Hall, New Jersey, USA, 2001.

[4] J. Banks, J. S. Carson, B. L. Nelson, D. M. Nicol, "Discrete-event System Simulation ( $3^{\text {rd }}$ Edition)", Prentice Hall, New Jersey, USA, 2000.

[5] J. Barr, "Gartner on open source: fair and insightful", 2004, available from http://trends.newsforge.com/. (accessed September, 2005).

[6] J. P. Bigus, J. Bigus, "Constructing Intelligent Agents with Java", John Wiley \& Sons Inc., Toronto, Canada, 1998.

[7] M. Bischop, "AppServer 4.5 Programmer's Guide for EJB", Borland Software Corporation, California, USA, 2001.

[8] G. Coulouris, J. Dollimore, T. Kindberg, "Distributed Systems: Concepts and Design (3rd Edition)", Addison Wesley, Boston, USA, 2000. 
[9] S. Crawford, "The Undercover Report: Conclusions and Comparisons (EJB Configuration)", 2003, available from http://www.scottcrawford.com. (accessed August, 2003).

[10] A. Diaconescu, A. Mos, J. Murphy, "Autonomic Performance Management in Component Based Software Systems", in Proceedings of the IEEE International Conference on Autonomic Computing, New York, USA, May 2004, pp. 214-221.

[11] E. Gamma, R. Helm, R. Johnson, J. Vlissides, "Design Patterns: Elements of Reusable Object-Oriented Software", Addison-Wesley Pearson Education, New Jersey, USA, 1994.

[12] A. G. Ganek, T. A. Corbi, "The Dawning of the Autonomic Computing Era", IBM Systems Journal, Vol. 42, No. 1, January 2003, pp. 5-18.

[13] J. Hunter, "Java Servlet Programming, Second Edition", O'Reilly \& Associates Inc., California, USA, 2001.

[14] R. K. Jain, "The Art of Computer System Performance Analysis", Wiley, New Jersey, USA, 1991.

[15] "JBoss Admin Development Guide (Release 3.0.7)", JBoss Inc., Atlanta, USA, 2003.

[16] D. S. Linthicum, "Enterprise Application Integration Addison-Wesley Information Technologies Series", Addison-Wesley, Boston, USA, 1999.

[17] Y. Liu, A. Fekete, I. Gorton, "Design-Level Performance Prediction of Component-Based Applications", IEEE Transactions Software Engineering, Vol. 31, No. 11, November 2005, pp. 928-941. 
[18] D. Menasce, F. Ribeiro, V. Almeida, R. Fonseca, "In Search of Invariants for Ebusiness Workloads", in Proceedings of $2^{\text {nd }} A C M$ Conference on Electronic Commerce, Minneapolis, USA, October 2000, pp. 17-20.

[19] D. A. Menascé, D. Barbará, R. Dodge, "Preserving QoS of E-commerce Sites through Self-Tuning: A Performance Model Approach", in Proceedings of 2001 ACM Conference on Electronic Commerce, Tampa, USA, October 2001, pp. 224-234.

[20] “OMG - The Common Object Request Broker: Architecture and Specification (Rev. 3)", Object Management Group, Needham, USA, 2002.

[21] D. S. Platt, "Introducing Microsoft .Net", Microsoft Press, California, USA, 2003.

[22] M. Raghavachari, D. Reimer, R. D. Johnson, “The Deployer's problem: Configuring Application Servers for Performance and Reliability", IBM Press, Toronto, Canada, 2005.

[23] M. Raghavachari, D. Reimer, R. D. Johnson, "The Deployer's Problem: Configuring Application Servers for Performance and Reliability", in Proceedings of the 25th International Conference on Software Engineering, Portland, USA, February 2003, pp. 284-489.

[24] E. Roman, R. P. Sriganesh, G. Brose, "Mastering Enterprise JavaBeans (3rd Edition)", Wiley, Indianapolis, USA, 2004.

[25] L. W. Russell, S. P. Morgan, E. G. Chron, "Clockwork: A new movement in autonomic systems", IBM Systems Journal, Vol. 42, No. 1, January 2003, pp. 77-84. 
[26] P. G. Sarang, K. Gabhart, A. Tost, T. McAllister, R. Adatia, M. Juric, T. Osborne, F. Arni, J. Lott, V. Nagarajan, C. A. Berry, D. O'Connor, J. Griffin, A. Mulder, D. Young, "Professional EJB”, Wrox Press, Birmingham, USA, 2001.

[27] A. Skonnard, "Understanding SOAP", 2002, available from http://msdn.microsoft.com/library/default.asp?url=/library/enus/dnsoap/htm1/understandsoap.asp. (accessed March, 2003).

[28] "Sun: Java Server Pages Specification v2.3", 2000, available from http://java.sun.com/products/isp. (accessed April 2001).

[29] "Sun: JavaTM 2 Platform Enterprise Edition Specification v 1.3", 2000, available on http://java.sun.com/j2ee/download.html. (accessed July 2001).

[30] "Sun - JavaBeans Specification v 2.0 (Final Release)", Santa Clara, USA, 2001.

[31] A. S. Tanenbaum, M. V. Steen, "Distributed Systems: Principles and Paradigms", Prentice Hall, New Jersey, USA, 2002.

[32] "Weblogic Programming Enterprise JavaBeans (Release 8.1)", Weblogic, San Jose, USA, 2006.

[33] "Whatis Online IT dictionary", 2004, available from http://www.whatis.com. (accessed November, 2004).

[34] S. L. Williams, "Application and performance management techniques for J2EE", 2004, available from http://devresource.hp.com. (accessed October 2004).

[35] R. H. Zakon, "Hobbes' Internet Timeline", 2005, available from http://www.zakon.org/robert/internet/timeline. (accessed March 2005). 
[36] Y. Zhang, A. Liu, W. Qu, "Comparing Industry Benchmarks for J2EE Application Server: IBM's Trade2 vs Sun's Ecperf', in Proceedings of the 26th Australian Computer Science Conference, Adelaide, Australia, February 2003, pp. 199-206. 


\section{Appendix A: Detailed Design of the URL Performance Analyzer}

Figure 26 illustrates the UML class diagram for our URL Performance Analyzer application.

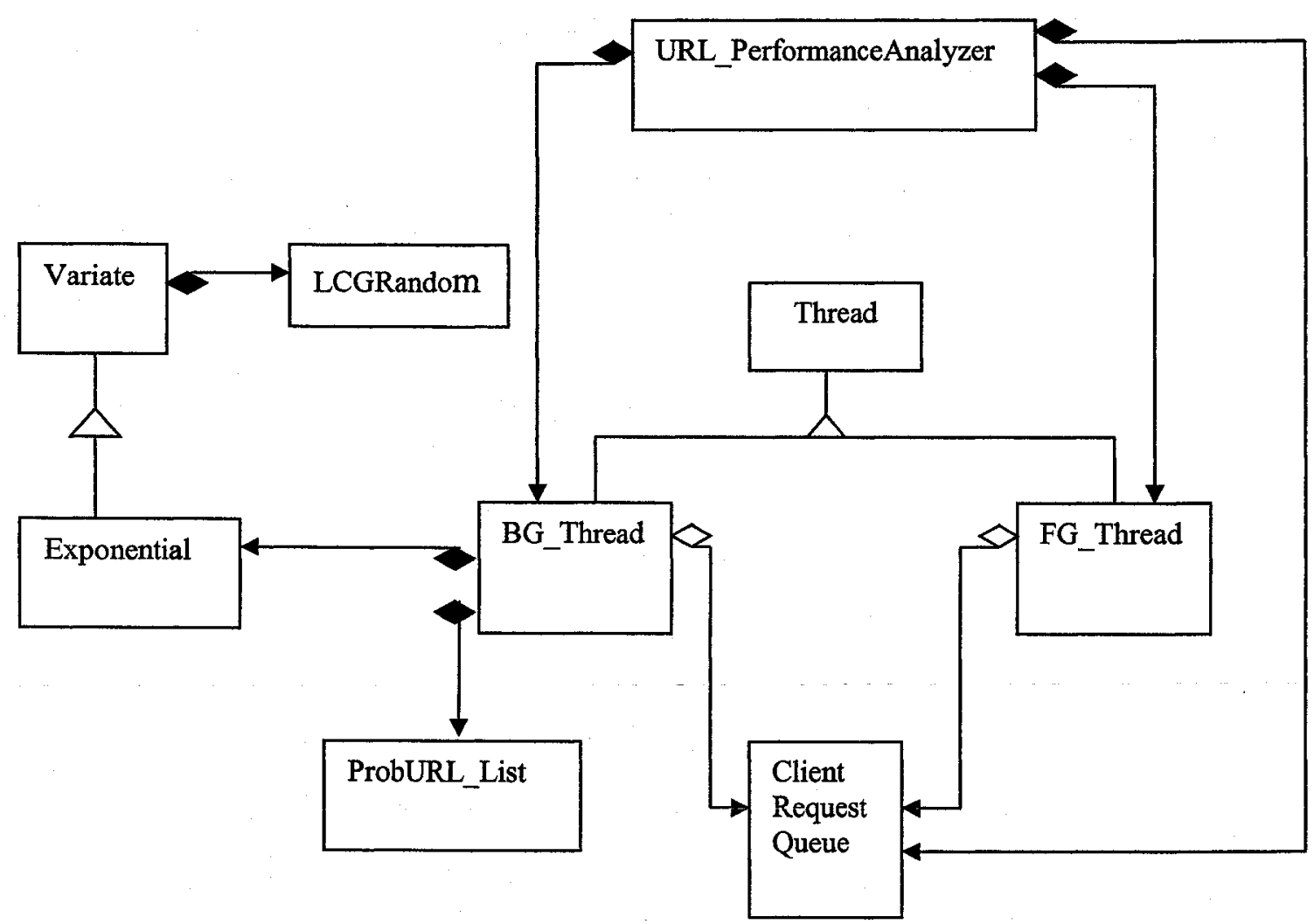

Figure 26: URL Performance Analyzer UML Class Model

The responsibility of each class is described.

- URL_PerformanceThread class:

This is the main thread that will create each of the following objects

ClientRequestQueue, BG_Thread, and FG_Thread

Since this is the main thread of the application, it reads a number of arguments from the Command Line Interface (CLI). 
CLI format:

$>$ java URL_PerformanceAnalyzer $<$ Arg1 $><\operatorname{Arg} 2><$ Arg3 $><\operatorname{Arg} 4>$

Where:

- Arg0: Application switch

- Arg1: This is the URL database path/file name.

- Arg2: This is the number of (simulated clients) foreground threads (FG_Thread Objects) to be created.

- Arg3: This is the number of requests to be generated in ClientRequestQueue.

- Arg4: This is the millisecond value for the client's request mean arrival time into the client request queue (ClientRequestQueue Object).

- ClientRequestQueue:

This is the client request queue class where HTTP client requests are queued as they arrive from the background thread (BG_Thread object), and are picked up (based on FIFO) by the foreground thread(s) (FG_Thread Object(s)).

$\operatorname{Read} /$ Write operations (methods) to the queue are synchronized, to ensure that only one thread has access to the queue at a particular time.

- FG_Thread:

This is the foreground thread of this application. One or multiple threads of this kind may run at the same time, as specified by the user/admin of this application.

The thread picks an HTTP request from the client queue (ClientRequestQueue Object) and initiates it. The response time for each URL request sent by this 
thread is measured, and stored within a statically ordered list of overall response times obtained from all running threads of this type

At the end of an experiment, the last running thread of this type would then proceed to perform the required experiment analysis of the stored list of overall response times. Analyses include transient removal and overall mean response time calculations.

- BG_Thread:

This is the background thread of this application, which creates the probabilistic URL list (ProbURL_List object), described in Section 4.1.1, and then based on a specified request arrival rate, selects a URL address from the list to insert/add into the client request queue (ClientRequestQueue Object).

This class also creates Exponential class instance(s) that are used to obtain exponentially distributed request inter-arrival times, as well as exponentially distributed request service times (if required).

- ProbURL_List:

This class is responsible for loading the URL data from the specified URL database file into its memory list (array). This memory list is constructed in a random, probabilistic fashion (as explained Section 4.1.1). Each line read from the URL database file contains a URL (representing a desired HTTP request to be generated) and its probability. Each read URL is then inserted into the memory list randomly and as often as specified by its probability. 
This class provides a read method that is used by the BG_Thread object. This read method returns a URL that is randomly selected and removed from the probabilistic URL list (ProbURL_List object) that was created earlier. 